\title{
Supersymmetric DBI equations in diverse dimensions from the BRS invariance of a pure spinor superstring
}

\author{
Sota Hanazawa* and Makoto Sakaguchi ${ }^{\dagger}$ \\ Department of Physics, Ibaraki University, Mito 310-8512, Japan
}

(Received 16 May 2019; published 5 August 2019)

\begin{abstract}
We examine the BRS invariance of the open pure spinor superstring in the presence of background superfields on a D p-brane. It is shown that the BRS invariance leads not only to boundary conditions on the spacetime spinors, but also to supersymmetric Dirac-Born-Infeld (DBI) equations of motion for the background superfields on the $\mathrm{D} p$-brane. These DBI equations are consistent with the supersymmetric DBI equations for a D9-brane.
\end{abstract}

DOI: 10.1103/PhysRevD.100.046006

\section{INTRODUCTION}

Dirac-Born-Infeld (DBI) theory is known as a nonlinear generalization of Maxwell theory and may describe, along with the Wess-Zumino action, the low-energy effective dynamics on a single D-brane in string theory. The bosonic DBI action is derived from the world-sheet analysis of the bosonic open string [1]. A supersymmetric DBI action should be a part of the effective action on a D-brane in type II superstring theory. In the Ramond-Neveu-Schwarz (RNS) formulation, however, it is difficult to read off the target space geometry coupling to Ramond-Ramond fields, because space-time supersymmetry becomes manifest only after the Gliozzi-Scherk-Olive projection. So the RNS superstring has led to the only bosonic sector of the supersymmetric DBI action [2].

The Green-Schwarz (GS) formulation has an advantage in this direction. The Wess-Zumino term which ensures the $\kappa$-invariance of the world-volume action of a D-brane is constructed in [3]. In [4], the $\kappa$-symmetric approach, socalled the superembedding formalism [5], is shown to lead to linearized supersymmetric DBI equations of motion for a D9-brane, which have the ten-dimensional $\mathcal{N}=2$ supersymmetry. Furthermore, in [6], the classical $\kappa$-invariance of an open GS superstring in an Abelian background is shown to imply that the background fields should satisfy full nonlinear equations of motion for a supersymmetric DBI action. The non-Abelian extension of this formalism is discussed in [7] as the boundary fermion formalism where

\footnotetext{
s.hanazawa.aq.09@gmail.com

${ }^{\dagger}$ makoto.sakaguchi.phys@vc.ibaraki.ac.jp
}

Published by the American Physical Society under the terms of the Creative Commons Attribution 4.0 International license. Further distribution of this work must maintain attribution to the author(s) and the published article's title, journal citation, and DOI. Funded by SCOAP ${ }^{3}$.
Chan-Patton factors describing coincident D-branes are replaced by boundary fermions. ${ }^{1}$

Unlike the formulations mentioned above, the pure spinor formulation [23] enables us to quantize a superstring in a super-Poincaré covariant manner. In this formulation, the $\kappa$-symmetry in the GS formulation is replaced with the BRS symmetry. It is shown in [24], correspondingly to the $\kappa$-symmetry analysis [6], that the classical BRS invariance of an open pure spinor superstring leads to supersymmetric DBI equations of motion on a D9-brane, which have the nonlinear $\mathcal{N}=1$ supersymmetry as well as the manifest $\mathcal{N}=1$ supersymmetry. These equations precisely coincide with those obtained in the superembedding formalism [25]. Furthermore, the non-Abelian extension of supersymmetric DBI equations is proposed. In [26] (see also [27,28]), D-brane boundary states are constructed in the pure spinor formulation. Especially, calculating the disk scattering amplitude suggests that the coupling of the boundary state to the background fields will reproduce the DBI kinetic term and the Wess-Zumino term of the D9-brane effective action. These achievements might imply the fact that the low-energy effective theory on the D9-brane is determined uniquely by the ten-dimensional $\mathcal{N}=2$ supersymmetry.

In this paper we will derive supersymmetric DBI equations of motion on a $\mathrm{D} p$-brane, as well as a D9-brane, from the BRS invariance of the open pure spinor superstring. Our approach is similar to that taken in [24] for a D9-brane. However the inclusion of Dirichlet components requires improvements which are not just a dimensional reduction of the case of a D9-brane. As in [24], we will provide two boundary terms, the counterterm $S_{\mathrm{b}}$ for the $\mathcal{N}=1$ supersymmetry transformation of the world-sheet action $S_{0}$ and the background superfield coupling $V$ as a

\footnotetext{
${ }^{1}$ Other than this study, there have been many attempts to extend to the non-Abelian DBI theory based on various approaches [8-22].
} 
relevant extension of the pure spinor vertex operator. It is found that the contribution of Dirichlet components in them cannot be determined unless considering the BRS invariance. In [24], by using nontrivial boundary conditions given by the general variation of $S_{0}+S_{\mathrm{b}}+V$, the BRS charge conservation leads to supersymmetric DBI equations for the D9-brane. On the other hand, we will show that supersymmetric DBI equations in diverse dimensions are extracted only from the BRS transformation of $S_{0}+S_{\mathrm{b}}+V$ under an identification on the D-brane position.

This paper is organized as follows. After introducing the type II pure spinor open superstring action in Sec. II, we construct the boundary term for the $\mathcal{N}=1$ supersymmetry invariance of this action in Sec. III. In Sec. IVA, background superfield coupling is found by considering the modification of a vertex operator in the open pure spinor superstring. In Sec. IV B, we confirm that these background superfields satisfy supersymmetric DBI equations of motion. The last section is devoted to summary and discussions. In addition, we give a brief review of the covariant approach for the ten-dimensional $\mathcal{N}=1$ superYang-Mills theory in Appendix A. We will formulate a vertex operator in the open pure spinor superstring in Appendix B. We show that our result can be derived also from improving the method used in [24] to include Dirichlet components in Appendix C.

\section{OPEN PURE SPINOR SUPERSTRING}

The world-sheet action of the type II pure spinor open superstring [23] is given as

$$
S_{0}=\frac{1}{\pi \alpha^{\prime}} \int \mathrm{d} z \mathrm{~d} \bar{z}\left\{\frac{1}{2} \partial x^{m} \bar{\partial} x_{m}+p_{\alpha} \bar{\partial} \theta^{\alpha}+\hat{p}_{\alpha} \partial \hat{\theta}^{\alpha}+\omega_{\alpha} \bar{\partial} \lambda^{\alpha}+\hat{\omega}_{\alpha} \partial \hat{\lambda}^{\alpha}\right\},
$$

where $x^{m}(m=0,1, \ldots, 9)$ is a ten-dimensional coordinate, $\theta^{\alpha}$ and $\hat{\theta}^{\alpha}(\alpha=1, \ldots, 16)$ are left- and right-moving tendimensional Majorana-Weyl spinors, respectively, and $\lambda^{\alpha}$ and $\hat{\lambda}^{\alpha}$ are bosonic ghosts satisfying pure spinor constrains $\lambda \gamma^{m} \lambda=\hat{\lambda} \gamma^{m} \hat{\lambda}=0$. The $\left(p_{\alpha}, \hat{p}_{\alpha}\right)$ and $\left(\omega_{\alpha}, \hat{\omega}_{\alpha}\right)$ are conjugate to $\left(\theta^{\alpha}, \hat{\theta}^{\alpha}\right)$ and $\left(\lambda^{\alpha}, \hat{\lambda}^{\alpha}\right)$, respectively. The world-sheet derivatives $\partial$ and $\bar{\partial}$ denote $\partial=\partial_{\tau}+\partial_{\sigma}$ and $\bar{\partial}=\partial_{\tau}-\partial_{\sigma}$, respectively. It implies $\mathrm{d} z \mathrm{~d} \bar{z}=-\frac{1}{2} \mathrm{~d} \tau \mathrm{d} \sigma$. The action is invariant under the gauge transformations $\delta_{\Lambda} \omega_{\alpha}=$ $\Lambda^{m}\left(\gamma_{m} \lambda\right)_{\alpha}$ and $\delta_{\hat{\Lambda}} \hat{\omega}_{\alpha}=\hat{\Lambda}^{m}\left(\gamma_{m} \hat{\lambda}\right)_{\alpha}$. We use $16 \times 16$ symmetric matrices $\gamma_{\alpha \beta}^{m}$ and $\gamma^{m \alpha \beta}$ which are off-diagonal blocks of the $32 \times 32$ gamma matrices and satisfy $\gamma_{\alpha \beta}^{m} \gamma^{n \beta \gamma}+\gamma_{\alpha \beta}^{n} \gamma^{m \beta \gamma}=2 \eta^{m n} \delta_{\alpha}^{\gamma}$. We frequently use the Fierz identity $\gamma_{m(\alpha \beta} \gamma_{\gamma) \delta}^{m}=0$.

The action (2.1) is invariant under the ten-dimensional $\mathcal{N}=2$ supersymmetry transformations,

$$
\begin{aligned}
& \delta_{\epsilon} \theta^{\alpha}=\epsilon^{\alpha}, \quad \delta_{\epsilon} \hat{\theta}^{\alpha}=\hat{\epsilon}^{\alpha}, \quad \delta_{\epsilon} x^{m}=\frac{1}{2} \theta \gamma^{m} \epsilon+\frac{1}{2} \hat{\theta} \gamma^{m} \hat{\epsilon}, \\
& \delta_{\epsilon} p_{\alpha}=\frac{1}{2} \partial x^{m}\left(\gamma_{m} \epsilon\right)_{\alpha}-\frac{1}{8}\left(\epsilon \gamma^{m} \theta\right)\left(\gamma_{m} \partial \theta\right)_{\alpha}, \quad \delta_{\epsilon} \hat{p}_{\alpha}=\frac{1}{2} \bar{\partial} x^{m}\left(\gamma_{m} \hat{\epsilon}\right)_{\alpha}-\frac{1}{8}\left(\hat{\epsilon} \gamma^{m} \hat{\theta}\right)\left(\gamma_{m} \bar{\partial} \hat{\theta}\right)_{\alpha},
\end{aligned}
$$

where parameters $\epsilon$ and $\hat{\epsilon}$ correspond to ten-dimensional Majorana-Weyl spinors. For an open superstring, we are left with a surface term,

$$
\delta_{\epsilon} S_{0}=\frac{1}{2 \pi \alpha^{\prime}} \int \mathrm{d} \tau\left\{\frac{1}{2}\left(\epsilon \gamma^{m} \theta-\hat{\epsilon} \gamma^{m} \hat{\theta}\right) \dot{x}_{m}+\frac{1}{12}\left(\epsilon \gamma^{m} \theta\right)\left(\theta \gamma_{m} \dot{\theta}\right)-\frac{1}{12}\left(\hat{\epsilon} \gamma^{m} \hat{\theta}\right)\left(\hat{\theta} \gamma_{m} \dot{\hat{\theta}}\right)\right\} \mid
$$

where "|" means "evaluated at the boundary" and we will omit it for brevity in the following. A dot on a field denotes the $\tau$-derivative of the field, while a prime does the $\sigma$ derivative. If there are no background fields, the surface term (2.3) can be eliminated by imposing usual boundary conditions for $\mathrm{D} p$-branes, ${ }^{2}$

\footnotetext{
${ }^{2}$ We must impose the same boundary condition on $\theta$ and $\lambda$ since BRS transformations relate them to each other. These boundary conditions also eliminate the surface term which comes from the BRS transformation of the world-sheet action $S_{0}$. See $[29,30]$ for related topics.
}

$x^{\prime \mu}=0, \quad \dot{x}^{i}=0, \quad \hat{\theta}=\gamma^{1 \cdots p} \theta, \quad \hat{\lambda}=\gamma^{1 \cdots p} \lambda$,

and $\mathcal{N}=1$ supersymmetry condition $\hat{\epsilon}=\gamma^{1 \cdots p} \epsilon$. These boundary conditions imply that $p=$ odd for the type IIB string while $p=$ even for the type IIA string. As usual, $x^{\mu}(\mu=0, \ldots, p)$ are Neumann coordinates, while $x^{i}(i=p+1, \ldots, 9)$ are Dirichlet coordinates.

Instead of imposing boundary conditions, we will consider coupling to the background superfields preserving the $\mathcal{N}=1$ supersymmetry specified by $\hat{\epsilon}=\gamma^{1 \cdots p} \epsilon$. To preserve 
$\mathcal{N}=1$ supersymmetry, we must introduce a boundary term which eliminates (2.3).

\section{III. $\mathcal{N}=1$ SUPERSYMMETRY AND BOUNDARY TERM}

Here we will introduce a boundary term $S_{\mathrm{b}}$ which leaves $S_{0}+S_{\mathrm{b}}$ invariant under the $\mathcal{N}=1$ supersymmetry. For this purpose, it is convenient to introduce the following objects,

$$
\begin{array}{ll}
\theta_{ \pm}^{\alpha}=\frac{1}{\sqrt{2}}\left(\hat{\theta}^{\alpha} \pm\left(\gamma^{1 \cdots p} \theta\right)^{\alpha}\right), & d_{\alpha}^{ \pm}=\sqrt{2}\left(\hat{d}_{\alpha} \pm\left(\gamma^{1 \cdots p} d\right)_{\alpha}\right), \\
\lambda_{ \pm}^{\alpha}=\frac{1}{\sqrt{2}}\left(\hat{\lambda}^{\alpha} \pm\left(\gamma^{1 \cdots p} \lambda\right)^{\alpha}\right), & \omega_{\alpha}^{ \pm}=\sqrt{2}\left(\hat{\omega}_{\alpha} \pm\left(\gamma^{1 \cdots p} \omega\right)_{\alpha}\right),
\end{array}
$$

where $d_{\alpha}=p_{\alpha}-\frac{1}{2} \partial x^{m}\left(\gamma_{m} \theta\right)_{\alpha}-\frac{1}{8}\left(\theta \gamma^{m} \partial \theta\right)\left(\gamma_{m} \theta\right)_{\alpha}$ and $\hat{d}_{\alpha}=$ $\hat{p}_{\alpha}-\frac{1}{2} \bar{\partial} x^{m}\left(\gamma_{m} \hat{\theta}\right)_{\alpha}-\frac{1}{8}\left(\hat{\theta} \gamma^{m} \bar{\partial} \hat{\theta}\right)\left(\gamma_{m} \hat{\theta}\right)_{\alpha}$ are invariant under the $\epsilon$ - and $\hat{\epsilon}$-supersymmetry in (2.2), respectively. By using these variables, the $\mathcal{N}=1$ supersymmetry transformations specified by $\hat{\epsilon}=\gamma^{1 \cdots p} \epsilon$ are represented as

$$
\begin{aligned}
\delta_{\eta} \theta_{+}^{\alpha} & =\eta^{\alpha}, \quad \delta_{\eta} \theta_{-}^{\alpha}=0, \quad \delta_{\eta} x^{\mu}=\frac{1}{2} \theta_{+} \gamma^{\mu} \eta, \\
\delta_{\eta} x^{i} & =\frac{1}{2} \theta_{-} \gamma^{i} \eta, \quad \delta_{\eta} \lambda_{ \pm}^{\alpha}=\delta_{\eta} \omega_{\alpha}^{ \pm}=0,
\end{aligned}
$$

where we introduced $\eta$ by $\eta \equiv \frac{1}{\sqrt{2}}\left(\hat{\epsilon}+\gamma^{1 \cdots p} \epsilon\right)$. The $\mathcal{N}=1$ supersymmetry transformation of $S_{0}$ is found to be

$$
\begin{aligned}
\delta_{\eta} S_{0}= & -\frac{1}{2 \pi \alpha^{\prime}} \int \mathrm{d} \tau\left\{\frac{1}{2}\left(\eta \gamma^{\mu} \theta_{-}\right) \dot{x}_{\mu}+\frac{1}{2}\left(\eta \gamma^{i} \theta_{+}\right) \dot{x}_{i}\right. \\
& \left.+\frac{1}{8}\left(\eta \gamma^{m} \theta_{+}\right)\left(\theta_{-} \gamma_{m} \dot{\theta}_{+}\right)+\frac{1}{24}\left(\eta \gamma^{m} \theta_{-}\right)\left(\theta_{-} \gamma_{m} \dot{\theta}_{-}\right)\right\},
\end{aligned}
$$

where we have used the Fierz identity.

The boundary term $S_{\mathrm{b}}$ we found is

$$
\begin{aligned}
S_{\mathrm{b}}= & \frac{1}{2 \pi \alpha^{\prime}} \int \mathrm{d} \tau\left\{\frac{1}{2} \Pi_{+}^{\mu}\left(\theta_{+} \gamma_{\mu} \theta_{-}\right)-\frac{1}{2} y^{i}\left(\theta_{+} \gamma_{i} \dot{\theta}_{+}\right)-\frac{1}{8}\left(\theta_{+} \gamma^{\mu} \theta_{-}\right)\left(\theta_{+} \gamma_{\mu} \dot{\theta}_{+}\right)+\frac{1}{8}\left(\theta_{+} \gamma^{i} \theta_{-}\right)\left(\theta_{+} \gamma_{i} \dot{\theta}_{+}\right)\right. \\
& \left.+\frac{1}{24}\left(\theta_{+} \gamma^{m} \theta_{-}\right)\left(\theta_{-} \gamma_{m} \dot{\theta}_{-}\right)+\frac{1}{2} c_{1} \Delta_{\alpha}^{+} \theta_{-}^{\alpha}+\frac{1}{2} c_{2} \omega_{\alpha}^{+} \lambda_{-}^{\alpha}+y_{i} \tilde{\Pi}_{+}^{i}\right\},
\end{aligned}
$$

where $c_{1}$ and $c_{2}$ are constants. We have introduced the following,

$$
\begin{array}{rlrl}
\Pi_{+}^{\mu} & =\frac{1}{2}\left(\hat{\Pi}^{\mu}+\Pi^{\mu}\right)-\frac{1}{2}\left(\theta_{-} \gamma^{\mu} \dot{\theta}_{-}\right), & \Pi_{-}^{i}=\frac{1}{2}\left(\hat{\Pi}^{i}+\Pi^{i}\right)-\frac{1}{2}\left(\theta_{-} \gamma^{i} \dot{\theta}_{+}\right), \\
\tilde{\Pi}_{-}^{\mu}=\frac{1}{2}\left(\hat{\Pi}^{\mu}-\Pi^{\mu}\right)-\frac{1}{2}\left(\theta_{-} \gamma^{\mu} \dot{\theta}_{+}\right), & \tilde{\Pi}_{+}^{i}=\frac{1}{2}\left(\hat{\Pi}^{i}-\Pi^{i}\right)-\frac{1}{2}\left(\theta_{-} \gamma^{i} \dot{\theta}_{-}\right), \\
y^{i} & =x^{i}+\frac{1}{2}\left(\theta_{+} \gamma^{i} \theta_{-}\right), \\
\Delta_{\alpha}^{+} & =d_{\alpha}^{+}+\frac{1}{2}\left(\gamma^{\mu} \theta_{-}\right)_{\alpha}\left(\hat{\Pi}_{\mu}-\Pi_{\mu}\right)+\frac{1}{2}\left(\gamma^{i} \theta_{-}\right)_{\alpha}\left(\hat{\Pi}_{i}+\Pi_{i}\right),
\end{array}
$$

where $\Pi^{m}=\partial x^{m}+\frac{1}{2} \theta \gamma^{m} \partial \theta$ and $\hat{\Pi}^{m}=\bar{\partial} x^{m}+\frac{1}{2} \hat{\theta} \gamma^{m} \bar{\partial} \hat{\theta}$ are $\epsilon$ - and $\hat{\epsilon}$-supersymmetry invariants, respectively. Objects in (3.5) are invariant under the $\mathcal{N}=1$ supersymmetry. To show this, we have to treat objects like $\theta_{ \pm}^{\prime}$ at the boundary. For this, we require that at the boundary

$$
\theta_{ \pm}^{\prime}=-\dot{\theta}_{\mp}, \quad \lambda_{ \pm}^{\prime}=-\dot{\lambda}_{\mp} .
$$

These are consistent with the bulk equations of motion $\bar{\partial} \theta^{\alpha}=\partial \hat{\theta}^{\alpha}=\bar{\partial} \lambda^{\alpha}=\partial \hat{\lambda}^{\alpha}=0$. It is shown that this choice leads to DBI equations in this paper. We also note that the last three terms in (3.4) are invariant under the $\mathcal{N}=1$ supersymmetry separately. This implies that they are not determined from the $\mathcal{N}=1$ supersymmetry. It is worth noting that (3.4) cannot be extracted as a dimensional reduction of the one for the D9-brane.

\section{A. BRS symmetry}

We shall show that the last term $y_{i} \tilde{\Pi}_{+}^{i}$ in (3.4) is required by the BRS invariance of $S_{0}+S_{\mathrm{b}}$, when there is no background superfield coupling.

The action (2.1) is invariant under a pair of BRS variations, say $\delta_{1}$ and $\delta_{2}$. In the presence of the boundary, these BRS variations must satisfy $\delta_{1}=\delta_{2}$ at the boundary. This implies that the BRS transformations $\delta_{Q}=\delta_{1}+\delta_{2}$ remain unbroken in the presence of the boundary, 


$$
\begin{aligned}
\delta_{Q} \theta_{ \pm}^{\alpha} & =\lambda_{ \pm}^{\alpha}, \quad \delta_{Q} \lambda_{ \pm}^{\alpha}=0, \quad \delta_{Q} \omega_{\alpha}^{ \pm}=d_{\alpha}^{ \pm}, \\
\delta_{Q} x^{\mu} & =\frac{1}{2} \lambda_{+} \gamma^{\mu} \theta_{+}+\frac{1}{2} \lambda_{-} \gamma^{\mu} \theta_{-}, \quad \delta_{Q} x^{i}=\frac{1}{2} \lambda_{+} \gamma^{i} \theta_{-}+\frac{1}{2} \lambda_{-} \gamma^{i} \theta_{+}, \quad \delta_{Q} y^{i}=\lambda_{+} \gamma^{i} \theta_{-}, \\
\delta_{Q} \Pi_{+}^{\mu}= & \lambda_{+} \gamma^{\mu} \dot{\theta}_{+}+\frac{1}{2} \lambda_{-} \gamma^{\mu} \dot{\theta}_{-}+\frac{1}{2} \dot{\lambda}_{-} \gamma^{\mu} \theta_{-}, \quad \delta_{Q} \Pi_{-}^{i}=\lambda_{+} \gamma^{i} \dot{\theta}_{-}+\frac{1}{2} \lambda_{-} \gamma^{i} \dot{\theta}_{+}+\frac{1}{2} \dot{\lambda}_{+} \gamma^{i} \theta_{-}, \\
\delta_{Q} \tilde{\Pi}_{-}^{\mu}= & \lambda_{+} \gamma^{\mu} \dot{\theta}_{-}+\frac{1}{2} \lambda_{-} \gamma^{\mu} \dot{\theta}_{+}+\frac{1}{2} \dot{\lambda}_{+} \gamma^{\mu} \theta_{-}, \quad \delta_{Q} \tilde{\Pi}_{+}^{i}=\lambda_{+} \gamma^{i} \dot{\theta}_{+}+\frac{1}{2} \lambda_{-} \gamma^{i} \dot{\theta}_{-}+\frac{1}{2} \dot{\lambda}_{-} \gamma^{i} \theta_{-}, \\
\delta_{Q} \Delta_{\alpha}^{+}= & -2\left(\gamma_{\mu} \lambda_{+}\right)_{\alpha} \Pi_{+}^{\mu}-2\left(\gamma_{i} \lambda_{+}\right)_{\alpha} \tilde{\Pi}_{+}^{i}-\left(\gamma_{\mu} \lambda_{-}\right)_{\alpha} \tilde{\Pi}_{-}^{\mu}-\left(\gamma_{i} \lambda_{-}\right)_{\alpha} \Pi_{-}^{i} \\
& -\left(\gamma^{m} \dot{\theta}_{-}\right)_{\alpha}\left(\lambda_{+} \gamma_{m} \theta_{-}\right)-\left(\gamma^{m} \theta_{-}\right)_{\alpha}\left(\lambda_{-} \gamma_{m} \dot{\theta}_{+}\right)-\frac{1}{2}\left(\gamma^{m} \lambda_{-}\right)_{\alpha}\left(\theta_{-} \gamma_{m} \dot{\theta}_{+}\right) .
\end{aligned}
$$

Again, we find the world-sheet action $S_{0}$ is BRS invariant $\delta_{Q} S_{0}=0$ up to a surface term, and satisfies

$$
\begin{aligned}
\delta_{Q}\left(S_{0}+S_{\mathrm{b}}\right)= & \frac{1}{2 \pi \alpha^{\prime}} \int \mathrm{d} \tau\left\{\left(1-c_{1}\right) \Pi_{+}^{\mu}\left(\lambda_{+} \gamma_{\mu} \theta_{-}\right)-\frac{1}{2}\left(c_{1}+c_{2}\right) \Pi_{-}^{i}\left(\lambda_{-} \gamma_{i} \theta_{-}\right)\right. \\
& -\frac{1}{2}\left(c_{1}+c_{2}\right) \tilde{\Pi}_{-}^{\mu}\left(\lambda_{-} \gamma_{\mu} \theta_{-}\right)+\left(1-c_{1}\right) \tilde{\Pi}_{+}^{i}\left(\lambda_{+} \gamma_{i} \theta_{-}\right) \\
& +\frac{1}{2}\left(c_{2}-c_{1}\right) \Delta_{\beta}^{+} \lambda_{-}^{\beta}+\left(-\frac{1}{3}+\frac{c_{1}+c_{2}}{4}\right)\left(\lambda_{-} \gamma^{m} \theta_{-}\right)\left(\dot{\theta}_{+} \gamma_{m} \theta_{-}\right) \\
& \left.+\frac{1}{2}\left(\frac{1}{3}-c_{1}\right)\left(\lambda_{+} \gamma^{m} \theta_{-}\right)\left(\theta_{-} \gamma_{m} \dot{\theta}_{-}\right)\right\} .
\end{aligned}
$$

Let us assume that there are no background fields. In this case, the (3.8) must be eliminated by the usual boundary conditions $\theta_{-}^{\alpha}=\lambda_{-}^{\alpha}=0$. It is obvious to see that these boundary conditions eliminate (3.8) as expected. It should be noted that this happens only when we include the term $y_{i} \tilde{\Pi}_{+}^{i}$ in (3.4).

Finally we comment on $y_{i}$. Remarkably, the BRS transformation of $S_{0}+S_{\mathrm{b}}$, at the boundary, is independent of $y_{i}$. More generally, we confirm $\delta\left(S_{0}+S_{\mathrm{b}}\right) / \delta y_{i} \mid=0$ in Appendix C. This strongly suggests that $y_{i}$ should represent the position of the $\mathrm{D} p$-brane.

\section{SUPERSYMMETRIC DBI EQUATIONS OF MOTION}

In this section, we will give the background coupling $V$ in terms of superfields on a D $p$-brane. Examining the BRS variation of $S_{0}+S_{\mathrm{b}}+V$, we obtain supersymmetric DBI equations of motion on the $\mathrm{D} p$-brane.

\section{A. Background superfield coupling for Dp-branes}

In Appendix $\mathrm{A}$, we define the ten-dimensional $\mathcal{N}=1$ superfield $A_{M}=\left(A_{m}, A_{\alpha}\right)$. We introduce background superfields on a $\mathrm{D} p$-brane as a dimensional reduction of $A_{M}: A_{m}=\left(A_{\mu}\left(x^{\mu}, \theta_{+}\right), A_{i}\left(x^{\mu}, \theta_{+}\right)\right)$and $A_{\alpha}=A_{\alpha}\left(x^{\mu}, \theta_{+}\right)$. Obviously they are invariant under the $\mathcal{N}=1$ supersymmetry. Similarly we introduce $\mathcal{W}^{\alpha}=\mathcal{W}^{\alpha}\left(x^{\mu}, \theta_{+}\right)$ and $F_{m n}=F_{m n}\left(x^{\mu}, \theta_{+}\right)$. We use the ten-dimensional Majorana-Weyl spinor notation throughout this paper. This means that we are considering the DBI equations with 16 supersymmetries, for example $\mathcal{N}=4$ supersymmetric DBI equations on a D3-brane.

The background coupling $V$ used in [24] is regarded as an extension of the vertex operator of the open pure spinor superstring. We give a brief review of the vertex operator in Appendix B.

The background coupling $V$ we introduce is

$$
\begin{aligned}
V= & \frac{1}{2 \pi \alpha^{\prime}} \int \mathrm{d} \tau\left\{\dot{\theta}_{+}^{\alpha} A_{\alpha}\left(x^{\mu}, \theta_{+}\right)+\Pi_{+}^{\mu} A_{\mu}\left(x^{\mu}, \theta_{+}\right)\right. \\
& +\tilde{\Pi}_{+}^{i} A_{i}\left(x^{\mu}, \theta_{+}\right)+\frac{1}{2} \Delta_{\alpha}^{+} \mathcal{W}^{\alpha}\left(x^{\mu}, \theta_{+}\right) \\
& \left.+\frac{1}{4} N_{+} \not{F}\left(x^{\mu}, \theta_{+}\right)\right\},
\end{aligned}
$$

where

$$
\begin{aligned}
\left(N_{+}\right)_{\alpha}^{\beta} & =\frac{1}{2} \omega_{\alpha}^{+} \lambda_{+}^{\beta}, \\
F_{\beta}^{\alpha} & =\delta_{\beta}^{\alpha} \mathcal{F}^{(0)}+\left(\gamma^{m n}\right)_{\beta}^{\alpha} \mathcal{F}_{m n}^{(2)}+\left(\gamma^{m n p q}\right)_{\beta}^{\alpha} \mathcal{F}_{m n p q}^{(4)} .
\end{aligned}
$$

Note that $\mathcal{F}^{(0)}, \mathcal{F}_{m n}^{(2)}$ and $\mathcal{F}_{m n p q}^{(4)}$ are some possible products of any number of vector field strengths 
$F_{m n}{ }^{3}$ which is consistent with analysis for D-brane boundary states [26] from the viewpoint of the pure spinor closed superstring. Needless to say, the $V$ is invariant under the $\mathcal{N}=1$ supersymmetry. Since we have made the factor $1 /\left(2 \pi \alpha^{\prime}\right)$ manifest in $V$, dimensions of these superfields differ from conventional ones. In this sense, we assign dimensions to $\left[A_{\alpha}\right],\left[A_{m}\right],\left[\mathcal{W}^{\alpha}\right]$ and $\left[F_{m n}\right]$ as $-\frac{3}{2},-1,-\frac{1}{2}$ and 0 , respectively.

\section{B. DBI equations from BRS symmetry}

In this subsection, we will add the background superfield coupling $V$ in (IVA) to the action $S_{0}+S_{\mathrm{b}}$ and then require that the BRS variation $\delta_{Q}\left(S_{0}+S_{\mathrm{b}}+V\right)$ vanishes. This requirement leads to boundary conditions on spacetime spinors and conditions on background superfields. The latter is found to be supersymmetric DBI equations of motion for them.

We find that the BRS variation $\delta_{Q} V$ may be expressed as

$$
\begin{aligned}
\delta_{Q} V= & \frac{1}{2 \pi \alpha^{\prime}} \int \mathrm{d} \tau\left\{\Pi_{+}^{\mu}\left[-\lambda_{+}^{\alpha} \partial_{\mu} A_{\alpha}+\lambda_{+}^{\alpha} D_{\alpha} A_{\mu}+\frac{1}{2}\left(\lambda_{-} \gamma^{n} \theta_{-}\right)\left(\partial_{n} A_{\mu}-\partial_{\mu} A_{n}\right)-\left(\lambda_{+} \gamma_{\mu} \mathcal{W}\right)\right]\right. \\
& +\Pi_{-}^{i}\left[-\frac{1}{2}\left(\lambda_{-} \gamma_{i} \mathcal{W}\right)-\frac{1}{8}\left(\gamma_{i} \theta_{-}\right)_{\alpha} \lambda_{+}^{\beta} \not^{\alpha}{ }_{\beta}\right]+\tilde{\Pi}_{-}^{\mu}\left[-\frac{1}{2}\left(\lambda_{-} \gamma_{\mu} \mathcal{W}\right)-\frac{1}{8}\left(\gamma_{\mu} \theta_{-}\right)_{\alpha} \lambda_{+}^{\beta} \mathscr{F}_{\beta}^{\alpha}\right] \\
& +\tilde{\Pi}_{+}^{i}\left[-\left(\lambda_{+} \gamma_{i} \mathcal{W}\right)+\lambda_{+}^{\alpha} D_{\alpha} A_{i}+\frac{1}{2}\left(\lambda_{-} \gamma^{\mu} \theta_{-}\right) \partial_{\mu} A_{i}\right]+\frac{1}{2} \Delta_{\beta}^{+}\left[-\lambda_{+}^{\alpha} D_{\alpha} \mathcal{W}^{\beta}-\frac{1}{2}\left(\lambda_{-} \gamma^{\mu} \theta_{-}\right) \partial_{\mu} \mathcal{W}^{\beta}+\frac{1}{4} \lambda_{+}^{\alpha} \not^{\beta}{ }_{\alpha}\right] \\
& +\frac{1}{4} N_{+\beta}^{\gamma}\left[\lambda_{+}^{\alpha} D_{\alpha} F^{\beta}{ }_{\gamma}+\frac{1}{2}\left(\lambda_{-} \gamma^{\mu} \theta_{-}\right) \partial_{\mu} \not^{\beta} \gamma_{\gamma}\right]+\dot{\theta}_{+}^{\beta}\left[-\lambda_{+}^{\alpha} D_{\beta} A_{\alpha}-\lambda_{+}^{\alpha} D_{\alpha} A_{\beta}-\frac{1}{2}\left(\lambda_{-} \gamma^{\mu} \theta_{-}\right) \partial_{\mu} A_{\beta}+\left(\gamma^{m} \lambda_{+}\right)_{\beta} A_{m}\right. \\
& \left.+\frac{1}{2}\left(\lambda_{-} \gamma^{m} \theta_{-}\right) D_{\beta} A_{m}+\frac{1}{2}\left(\gamma^{m} \lambda_{-}\right)_{\beta}\left(\theta_{-} \gamma_{m} \mathcal{W}\right)+\frac{1}{4}\left(\gamma^{m} \theta_{-}\right)_{\beta}\left(\lambda_{-} \gamma_{m} \mathcal{W}\right)+\frac{1}{16}\left(\gamma^{m} \theta_{-}\right)_{\beta}\left(\gamma_{m} \theta_{-}\right)_{\gamma} \lambda_{+}^{\alpha} \not{F}^{\gamma}{ }_{\alpha}\right] \\
& \left.+\dot{\theta}_{-}^{\beta}\left[-\frac{1}{2}\left(\lambda_{+} \gamma^{m} \theta_{-}\right)\left(\gamma_{m} \mathcal{W}\right)_{\beta}\right]\right\} .
\end{aligned}
$$

Note that the supercovariant derivative on the $\mathrm{D} p$-brane is defined by

$$
D_{\alpha}=\frac{\partial}{\partial \theta_{+}^{\alpha}}+\frac{1}{2}\left(\gamma^{\mu} \theta_{+}\right)_{\alpha} \partial_{\mu} .
$$

Gathering (3.8) and (4.4) together, we obtain the BRS variation of $S_{0}+S_{\mathrm{b}}+V$ as

$$
\delta_{Q}\left(S_{0}+S_{\mathrm{b}}+V\right)=\frac{1}{2 \pi \alpha^{\prime}} \int \mathrm{d} \tau\left\{\Pi_{+}^{\mu} \mathrm{X}_{\mu}+\tilde{\Pi}_{+}^{i} \mathrm{X}_{i}+\Pi_{-}^{i} \mathrm{Y}_{i}+\tilde{\Pi}_{-}^{\mu} \mathrm{Y}_{\mu}-\frac{1}{2} \Delta_{\beta}^{+} \Lambda^{\beta}+\frac{1}{4} N_{+\beta}{ }^{\alpha} \mathrm{Z}_{\alpha}^{\beta}+\dot{\theta}_{+}^{\alpha} \Theta_{\alpha}^{+}+\dot{\theta}_{-}^{\alpha} \Theta_{\alpha}^{-}\right\},
$$

where $\mathrm{X}_{m}, \mathrm{Y}_{m}, \Lambda^{\beta}, \mathrm{Z}_{\alpha}^{\beta}$ and $\Theta_{\alpha}^{ \pm}$are given as follows:

$$
\begin{gathered}
\mathrm{X}_{m} \equiv\left(1-c_{1}\right)\left(\lambda_{+} \gamma_{m} \theta_{-}\right)-\lambda_{+}^{\alpha} \partial_{m} A_{\alpha}+\lambda_{+}^{\alpha} D_{\alpha} A_{m}-\frac{1}{2}\left(\lambda_{-} \gamma^{n} \theta_{-}\right)\left(\partial_{m} A_{n}-\partial_{n} A_{m}\right)-\left(\lambda_{+} \gamma_{m} \mathcal{W}\right), \\
\mathrm{Y}_{m} \equiv-\frac{1}{2}\left(c_{1}+c_{2}\right)\left(\lambda_{-} \gamma_{m} \theta_{-}\right)-\frac{1}{2}\left(\lambda_{-} \gamma_{m} \mathcal{W}\right)-\frac{1}{8}\left(\gamma_{m} \theta_{-}\right)_{\alpha} \lambda_{+}^{\beta} \not{F}_{\beta}^{\alpha}, \\
\Lambda^{\beta} \equiv\left(c_{1}-c_{2}\right) \lambda_{-}^{\beta}+\lambda_{+}^{\alpha} D_{\alpha} \mathcal{W}^{\beta}+\frac{1}{2}\left(\lambda_{-} \gamma^{\mu} \theta_{-}\right) \partial_{\mu} \mathcal{W}^{\beta}-\frac{1}{4} \lambda_{+}^{\alpha} \not F^{\beta}{ }_{\alpha}, \\
Z_{\alpha}^{\beta} \equiv \lambda_{+}^{\gamma} D_{\gamma} \not^{\beta}{ }_{\alpha}+\frac{1}{2}\left(\lambda_{-} \gamma^{\mu} \theta_{-}\right) \partial_{\mu} \not \not^{\beta}{ }_{\alpha},
\end{gathered}
$$

${ }^{3}$ There are no more higher forms because of the property,

$$
\omega_{\alpha}\left(\gamma^{m_{1} \cdots m_{2 k}}\right)^{\alpha}{ }_{\beta} \lambda^{\beta}= \pm \frac{1}{(10-2 k) !}(-1)^{k+1} \epsilon^{m_{1} \cdots m_{2 k}}{ }_{n_{1} \cdots n_{10-2 k}} \omega_{\alpha}\left(\gamma^{n_{1} \cdots n_{10-2 k}}\right)^{\alpha}{ } \lambda^{\beta},
$$

where the sign in the right-hand side depends on the chirality of $\lambda$. 


$$
\begin{aligned}
& \Theta_{\alpha}^{+} \equiv\left(-\frac{1}{3}+\frac{c_{1}+c_{2}}{4}\right)\left(\gamma^{m} \theta_{-}\right)_{\alpha}\left(\lambda_{-} \gamma_{m} \theta_{-}\right)-\lambda_{+}^{\beta}\left(D_{\alpha} A_{\beta}+D_{\beta} A_{\alpha}\right)-\frac{1}{2}\left(\lambda_{-} \gamma^{\mu} \theta_{-}\right) \partial_{\mu} A_{\alpha} \\
&+\left(\gamma^{m} \lambda_{+}\right)_{\alpha} A_{m}+\frac{1}{2}\left(\lambda_{-} \gamma^{m} \theta_{-}\right) D_{\alpha} A_{m}+\frac{1}{2}\left(\gamma^{m} \lambda_{-}\right)_{\alpha}\left(\theta_{-} \gamma_{m} \mathcal{W}\right) \\
&+\frac{1}{4}\left(\gamma^{m} \theta_{-}\right)_{\alpha}\left(\lambda_{-} \gamma_{m} \mathcal{W}\right)+\frac{1}{16}\left(\gamma^{m} \theta_{-}\right)_{\alpha}\left(\gamma_{m} \theta_{-}\right)_{\gamma} \lambda_{+}^{\beta} F_{\beta}^{\gamma}, \\
& \Theta_{\alpha}^{-} \equiv \frac{1}{2}\left(c_{1}-\frac{1}{3}\right)\left(\gamma^{m} \theta_{-}\right)_{\alpha}\left(\lambda_{+} \gamma_{m} \theta_{-}\right)-\frac{1}{2}\left(\lambda_{+} \gamma^{m} \theta_{-}\right)\left(\gamma_{m} \mathcal{W}\right)_{\alpha} .
\end{aligned}
$$

In the following, we will examine conditions that each term in (4.6) vanishes.

To achieve our purpose, first, we focus on the term $\tilde{\Pi}_{+}^{i} \mathrm{X}_{i}$ in (4.6) which takes the form

$$
\tilde{\Pi}_{+}^{i}\left[-\lambda_{+}^{\alpha} \gamma_{i \alpha \beta}\left(c_{1} \theta_{-}^{\beta}+\mathcal{W}^{\beta}\right)+\delta_{Q}\left(y_{i}+A_{i}\right)\right]
$$

Here we assume that $\delta_{Q}\left(y_{i}+A_{i}\right)=0$. This follows from the fact that we fix degrees of freedom for the D-brane position by

$$
y_{i}=-A_{i}
$$

In fact, this identification turns to $y_{i}=0$ in the $\alpha^{\prime} \rightarrow 0$ limit after the scaling $A_{i} \rightarrow\left(2 \pi \alpha^{\prime}\right) A_{i}$. It implies that we consider a D-brane sitting at the origin. As we will see below, the BRS transformation of (4.14) turns into one of the DBI equations and the derivation of (4.14) with respect to the time-coordinate $\tau$ also turns into the Dirichlet boundary condition. One may add a constant to the right-hand side of (4.14) to consider a D-brane sitting outside the origin, but this will not affect the DBI equation and the Dirichlet boundary condition as anticipated. In addition, we obtain the boundary condition on $\theta_{-}$as

$$
\theta_{-}^{\beta}=-\frac{1}{c_{1}} \mathcal{W}^{\beta}
$$

This eliminates $\theta_{-}^{\alpha}$ from (4.6) completely. Hereafter we understand $\theta_{-}^{\alpha}$ as (4.15). Note that (4.15) also leads to

$$
\dot{\theta}_{-}^{\beta}=-\frac{1}{c_{1}}\left(\Pi_{+}^{\mu} \partial_{\mu} \mathcal{W}^{\beta}+\dot{\theta}_{+}^{\gamma} D_{\gamma} \mathcal{W}^{\beta}\right)
$$

Second, the terms $\Pi_{-}^{i} \mathrm{Y}_{i}$ and $\tilde{\Pi}_{-}^{\mu} \mathrm{Y}_{\mu}$ reduce to

$$
\Pi_{-}^{m}\left[\left(c_{2} \lambda_{-}^{\alpha}+\frac{1}{4} \lambda_{+}^{\beta} \mathscr{F}_{\beta}^{\alpha}\right) \frac{1}{c_{1}}\left(\gamma_{m} \mathcal{W}\right)_{\alpha}\right]
$$

and imply the boundary condition on $\lambda_{-}$,

$$
\lambda_{-}^{\alpha}=-\frac{1}{4 c_{2}} \lambda_{+}^{\beta} \not_{\beta}^{\alpha}
$$

This eliminates $\lambda_{-}^{\alpha}$ from (4.6) completely. Hereafter we understand $\lambda_{-}^{\alpha}$ as (4.18).

Here, it is better to comment on two consequences of the boundary conditions (4.15) and (4.18). First, consider the limit $\alpha^{\prime} \rightarrow 0$. The limit $\alpha^{\prime} \rightarrow 0$, after rescaling $A_{\alpha} \rightarrow$ $\left(2 \pi \alpha^{\prime}\right) A_{\alpha}, A_{m} \rightarrow\left(2 \pi \alpha^{\prime}\right) A_{m}, \mathcal{W}^{\alpha} \rightarrow\left(2 \pi \alpha^{\prime}\right) \mathcal{W}^{\alpha}$ and $F_{m n} \rightarrow$ $\left(2 \pi \alpha^{\prime}\right) F_{m n}$, turns the boundary conditions (4.15) and (4.18) to the usual boundary conditions $\theta_{-}^{\alpha}=\lambda_{-}^{\alpha}=0$. The BRS invariance $\delta_{Q}\left(S_{0}+S_{\mathrm{b}}+V\right)=0$ then implies $\delta_{Q} V=0$, since $\delta_{Q}\left(S_{0}+S_{\mathrm{b}}\right)=0$ under these boundary conditions. We can show that $\delta_{Q} V=0$ with usual boundary conditions leads to the super-Yang-Mills equations of motion (B9)(B11) as discussed in Appendix B.

We consider the BRS variation $\delta_{Q}\left(y_{i}+A_{i}\right)=0$. To evaluate it, we note that $\delta_{Q} A_{i}=\lambda_{+}^{\alpha} D_{\alpha} A_{i}+\frac{1}{2}\left(\lambda_{-} \gamma^{\mu} \theta_{-}\right) \partial_{\mu} A_{i}$. Under the boundary conditions (4.15) and (4.18), the equation $\delta_{Q}\left(y_{i}+A_{i}\right)=0$ is shown to reduce to the following equation:

$-D_{\alpha} A_{i}+\frac{1}{c_{1}}\left(\gamma_{i} \mathcal{W}\right)_{\alpha}-\frac{1}{8 c_{1} c_{2}} F_{\alpha}^{\beta}\left(\gamma^{\mu} \mathcal{W}\right)_{\beta} \partial_{\mu} A_{i}=0$

This is one of the DBI equations. Furthermore, noting that $\dot{A}_{i}=\dot{\theta}_{+}^{\alpha} D_{\alpha} A_{i}+\Pi_{+}^{\mu} \partial_{\mu} A_{i}$, one finds that the time derivative of (4.14) turns into the Dirichlet boundary condition given in $(\mathrm{C} 11)$.

Let us return to the subject. Third, the term $\Delta_{\beta}^{+} \Lambda^{\beta}$ in (4.6) is examined. We see that $\Lambda^{\beta}=0$ reduces to

$\frac{1}{c_{1}} D_{\alpha} \mathcal{W}^{\beta}-\frac{1}{4 c_{2}} \boldsymbol{F}^{\beta}{ }_{\alpha}+\frac{1}{8 c_{1}^{2} c_{2}} \not^{\gamma}{ }_{\alpha}\left(\gamma^{\mu} \mathcal{W}\right)_{\gamma} \partial_{\mu} \mathcal{W}^{\beta}=0$

This is one of the DBI equations on a D p-brane. This equation ensures that conditions (4.15) and (4.18) are consistent with BRS transformations $\delta_{Q} \theta_{-}^{\alpha}=\lambda_{-}^{\alpha}$ and $\delta_{Q} \lambda_{-}^{\alpha}=0$. 
As was done in [24], it is convenient to introduce a covariant derivative $\hat{D}_{\alpha}$ by

$$
\hat{D}_{\alpha} \equiv D_{\alpha}+\frac{1}{8 c_{1} c_{2}} \mathbb{F}_{\alpha}^{\gamma}\left(\gamma^{\mu} \mathcal{W}\right)_{\gamma} \partial_{\mu}
$$

Applying it to $\frac{1}{c_{1}} \mathcal{W}^{\beta}$, we obtain

$\frac{1}{c_{1}} \hat{D}_{\alpha} \mathcal{W}^{\beta}=\frac{1}{c_{1}} D_{\alpha} \mathcal{W}^{\beta}+\frac{1}{8 c_{1}^{2} c_{2}} F_{\alpha}^{\gamma}\left(\gamma^{\mu} \mathcal{W}\right)_{\gamma} \partial_{\mu} \mathcal{W}^{\beta}=\frac{1}{4 c_{2}} F_{\alpha}^{\beta}$,

where in the last equality (4.20) is used. On the other hand, as (4.20) implies

$\frac{1}{4 c_{2}} \mathscr{F}^{\beta}{ }_{\alpha}=\frac{1}{c_{1}} D_{\alpha} \mathcal{W}^{\gamma}\left(\delta_{\beta}^{\gamma}-\frac{1}{2 c_{1}^{2}}\left(\gamma^{\mu} \mathcal{W}\right)_{\beta} \partial_{\mu} \mathcal{W}^{\gamma}\right)^{-1}$

$\hat{D}_{\alpha}$ is expressed as

$\hat{D}_{\alpha}=D_{\alpha}+\frac{1}{2 c_{1}^{2}} D_{\alpha} \mathcal{W}^{\delta}\left(\delta_{\gamma}^{\delta}-\frac{1}{2 c_{1}^{2}}\left(\gamma^{\nu} \mathcal{W}\right)_{\gamma} \partial_{\nu} \mathcal{W}^{\delta}\right)^{-1}\left(\gamma^{\mu} \mathcal{W}\right)_{\gamma} \partial_{\mu}$

It follows that it satisfies the following anticommutation relation:

$$
\begin{aligned}
\left\{\hat{D}_{\alpha}, \hat{D}_{\beta}\right\}= & \left(\gamma_{\alpha \beta}^{\mu}+\frac{1}{16 c_{2}^{2}} \not{ }_{\alpha}{ }_{\alpha} \not_{\beta}^{\delta} \gamma_{\gamma \delta}^{\mu}\right) \hat{\partial}_{\mu} \\
\hat{\partial}_{\mu} \equiv & \partial_{\mu}+\frac{1}{2 c_{1}^{2}} \partial_{\mu} \mathcal{W}^{\alpha}\left(\delta_{\beta}^{\alpha}-\frac{1}{2 c_{1}^{2}} \gamma_{\beta \gamma}^{\nu} \mathcal{W}^{\gamma} \partial_{\nu} \mathcal{W}^{\alpha}\right)^{-1} \\
& \times\left(\gamma^{\rho} \mathcal{W}\right)_{\beta} \partial_{\rho} .
\end{aligned}
$$

Fourth, the term $\left(N_{+}\right)_{\beta}^{\alpha} Z^{\beta}{ }_{\alpha}$ in (4.6) is examined. Using (4.22) and (4.25), it turns to

$$
\begin{aligned}
\lambda_{+}^{\alpha} \lambda_{+}^{\gamma}\left(\frac{1}{c_{2}} \hat{D}_{\alpha} \not^{\beta}{ }_{\gamma}\right)= & \lambda_{+}^{\alpha} \lambda_{+}^{\gamma}\left(\frac{4}{c_{1}} \hat{D}_{\alpha} \hat{D}_{\gamma} \mathcal{W}^{\beta}\right) \\
= & \lambda_{+}^{\alpha} \lambda_{+}^{\gamma}\left(\gamma_{\alpha \gamma}^{\mu}+\frac{1}{16 c_{2}^{2}} \not_{\alpha}^{\delta} \not^{\eta}{ }_{\gamma} \gamma_{\delta \eta}^{\mu}\right) \\
& \times \frac{2}{c_{1}} \hat{\partial}_{\mu} \mathcal{W}^{\beta},
\end{aligned}
$$

which vanishes due to the pure spinor constraint $\lambda_{+} \gamma^{\mu} \lambda_{+}+\lambda_{-} \gamma^{\mu} \lambda_{-}=0$.

Fifth, we consider terms including $\Pi_{+}^{\mu}$ in (4.6), $\Pi_{+}^{\mu} \mathrm{X}_{\mu}-$ $\frac{1}{c_{1}} \Pi_{+}^{\mu} \partial_{\mu} \mathcal{W}^{\alpha} \Theta_{\alpha}^{-}$, where the second term comes from (4.16). It is straightforward to see that it is eliminated by

$$
\begin{gathered}
\partial_{\mu} A_{\alpha}-D_{\alpha} A_{\mu}+\frac{1}{c_{1}}\left(\gamma_{\mu} \mathcal{W}\right)_{\alpha}+\frac{1}{6 c_{1}^{3}}\left(\gamma^{n} \mathcal{W}\right)_{\alpha}\left(\mathcal{W} \gamma_{n} \partial_{\mu} \mathcal{W}\right) \\
+\frac{1}{8 c_{1} c_{2}} \not^{\beta}{ }_{\alpha}\left(\gamma^{n} \mathcal{W}\right)_{\beta}\left(\partial_{\mu} A_{n}-\partial_{n} A_{\mu}\right)=0
\end{gathered}
$$

which is one of the DBI equations. Combining it with (4.19), we obtain

$$
\begin{gathered}
\partial_{m} A_{\alpha}-D_{\alpha} A_{m}+\frac{1}{c_{1}}\left(\gamma_{m} \mathcal{W}\right)_{\alpha}+\frac{1}{6 c_{1}^{3}}\left(\gamma^{n} \mathcal{W}\right)_{\alpha}\left(\mathcal{W} \gamma_{n} \partial_{m} \mathcal{W}\right) \\
+\frac{1}{8 c_{1} c_{2}} F^{\beta}{ }_{\alpha}\left(\gamma^{n} \mathcal{W}\right)_{\beta}\left(\partial_{m} A_{n}-\partial_{n} A_{m}\right)=0
\end{gathered}
$$

Finally, we consider terms including $\dot{\theta}_{+}^{\alpha}$ in (4.6), $\dot{\theta}_{+}^{\alpha} \Theta_{\alpha}^{+}-\frac{1}{c_{1}} \dot{\theta}_{+}^{\beta} D_{\beta} \mathcal{W}^{\alpha} \Theta_{\alpha}^{-}$, where the second term comes from (4.16). These terms are eliminated by

$$
\begin{aligned}
- & D_{\alpha} A_{\beta}-D_{\beta} A_{\alpha}+\gamma_{\alpha \beta}^{m} A_{m}-\frac{1}{6 c_{1}^{3}}\left(\gamma^{m} \mathcal{W}\right)_{\alpha}\left(\mathcal{W} \gamma_{m} D_{\beta} \mathcal{W}\right) \\
& +\frac{1}{12 c_{1}^{2} c_{2}} F^{\gamma}{ }_{\alpha}\left(\gamma^{m} \mathcal{W}\right)_{\beta}\left(\gamma_{m} \mathcal{W}\right)_{\gamma} \\
& -\frac{1}{8 c_{1} c_{2}} F_{\alpha}^{\gamma}\left(\gamma^{m} \mathcal{W}\right)_{\gamma}\left(\partial_{m} A_{\beta}-D_{\beta} A_{m}\right)=0
\end{aligned}
$$

By eliminating $\partial_{m} A_{\beta}-D_{\beta} A_{m}$ by (4.28), it reduces to

$$
\begin{aligned}
- & D_{\alpha} A_{\beta}-D_{\beta} A_{\alpha}+\gamma_{\alpha \beta}^{m} A_{m}-\frac{1}{6 c_{1}^{3}}\left(\gamma^{m} \mathcal{W}\right)_{\alpha}\left(\mathcal{W} \gamma_{m} D_{\beta} \mathcal{W}\right) \\
& +\frac{1}{6 c_{1}^{2}}\left(\gamma^{m} \mathcal{W}\right)_{\beta}\left(\gamma_{m} \mathcal{W}\right)_{\gamma} \\
& \times\left\{-\frac{1}{4 c_{2}} F_{\alpha}^{\gamma}+\frac{1}{8 c_{1}^{2} c_{2}} \mathscr{F}_{\alpha}^{\delta}\left(\gamma^{n} \mathcal{W}\right)_{\delta} \partial_{n} \mathcal{W}\right\} \\
& +\frac{1}{64 c_{1}^{2} c_{2}^{2}} \mathscr{W}_{\alpha}^{\gamma} \not_{\beta}^{\delta}\left(\gamma^{m} \mathcal{W}\right)_{\gamma}\left(\gamma^{n} \mathcal{W}\right)_{\delta}\left(\partial_{m} A_{n}-\partial_{n} A_{m}\right)=0 .
\end{aligned}
$$

Finally substituting (4.20) into the expression in the curly braces in (4.30), we obtain

$$
\begin{aligned}
& -D_{\alpha} A_{\beta}-D_{\beta} A_{\alpha}+\gamma_{\alpha \beta}^{m} A_{m}-\frac{1}{6 c_{1}^{3}}\left(\gamma^{m} \mathcal{W}\right)_{\alpha}\left(\mathcal{W} \gamma_{m} D_{\beta} \mathcal{W}\right) \\
& -\frac{1}{6 c_{1}^{3}}\left(\gamma^{m} \mathcal{W}\right)_{\beta}\left(\mathcal{W} \gamma_{m} D_{\alpha} \mathcal{W}\right) \\
& +\frac{1}{64 c_{1}^{2} c_{2}^{2}} F_{\alpha}^{\gamma} \digamma^{\delta}{ }_{\beta}\left(\gamma^{m} \mathcal{W}\right)_{\gamma}\left(\gamma^{n} \mathcal{W}\right)_{\delta}\left(\partial_{m} A_{n}-\partial_{n} A_{m}\right)=0 .
\end{aligned}
$$

As a result, we have obtained not only boundary conditions (4.15) and (4.18), but also independent equations for background superfields (4.28), (4.31) and (4.20) which eliminates (4.6). We note that $c_{1}$ and $c_{2}$ can be absorbed into redefinitions of $\mathcal{W}^{\alpha}$ and $F^{\beta}{ }_{\alpha}$ as $\frac{1}{c_{1}} \mathcal{W}^{\alpha} \rightarrow \mathcal{W}^{\alpha}$ 
and $\frac{1}{c_{2}} \not{F}_{\alpha}^{\beta} \rightarrow \not{F}^{\beta}{ }_{\alpha}$. So we will set $c_{1}=c_{2}=1$ without loss of generality. ${ }^{4}$

Summarizing, we have obtained supersymmetric DBI equations of motion on a $\mathrm{D} p$-brane:

$$
\begin{gathered}
\partial_{m} A_{\alpha}-D_{\alpha} A_{m}+\left(\gamma_{m} \mathcal{W}\right)_{\alpha}+\frac{1}{6}\left(\gamma^{n} \mathcal{W}\right)_{\alpha}\left(\mathcal{W} \gamma_{n} \partial_{m} \mathcal{W}\right) \\
+\frac{1}{8} \not^{\beta}{ }_{\alpha}\left(\gamma^{n} \mathcal{W}\right)_{\beta}\left(\partial_{m} A_{n}-\partial_{n} A_{m}\right)=0, \\
D_{\alpha} A_{\beta}+D_{\beta} A_{\alpha}-\gamma_{\alpha \beta}^{m} A_{m}+\frac{1}{6}\left(\gamma^{m} \mathcal{W}\right)_{\alpha}\left(\mathcal{W} \gamma_{m} D_{\beta} \mathcal{W}\right) \\
+\frac{1}{6}\left(\gamma^{m} \mathcal{W}\right)_{\beta}\left(\mathcal{W} \gamma_{m} D_{\alpha} \mathcal{W}\right) \\
-\frac{1}{64} \not{ }^{\gamma}{ }_{\alpha} \not^{\delta}{ }_{\beta}\left(\gamma^{m} \mathcal{W}\right)_{\gamma}\left(\gamma^{n} \mathcal{W}\right)_{\delta}\left(\partial_{m} A_{n}-\partial_{n} A_{m}\right)=0, \\
D_{\alpha} \mathcal{W}^{\beta}-\frac{1}{4} \not^{\beta}{ }_{\alpha}+\frac{1}{8} \not F^{\gamma}{ }_{\alpha}\left(\gamma^{\mu} \mathcal{W}\right)_{\gamma} \partial_{\mu} \mathcal{W}^{\beta}=0 .
\end{gathered}
$$

In the last equation, the index $\mu$ may be replaced with $m$ because $\partial_{i} \mathcal{W}^{\beta}=0$. Now it is manifest that our DBI equations on a $\mathrm{D} p$-brane can be expressed in a tendimensional covariant fashion. In other words, our result coincides with the dimensional reduction of those for a D9-brane, though the ten-dimensional covariance was absent in the beginning of our analysis.

\section{SUMMARY AND DISCUSSIONS}

We have examined the BRS invariance of the open pure spinor superstring in the presence of background superfields on a D $p$-brane. It was shown that the BRS invariance leads not only to boundary conditions on the spacetime spinors, but also to supersymmetric DBI equations of motion for the background superfields on a $\mathrm{D} p$-brane. These DBI equations precisely coincide with those obtained by a dimensional reduction of the supersymmetric DBI equations for the Abelian D9-brane given in [24,25].

We have introduced the boundary term $S_{\mathrm{b}}$ and the background coupling $V$. Both are determined by the BRS symmetry. In fact, $S_{\mathrm{b}}$ was shown to satisfy $\delta_{Q}\left(S_{0}+S_{\mathrm{b}}\right)=0$, when we take the limit $\alpha^{\prime} \rightarrow 0$ and turn off the background couplings. As for $V$, we have shown that the conditions for $\delta_{Q}\left(S_{0}+S_{\mathrm{b}}+V\right)=0$ reduce to the dimensional reduction of the super-Yang-Mills equations when $\alpha^{\prime} \rightarrow 0$. In fact, taking the limit $\alpha^{\prime} \rightarrow 0$, after rescaling $A_{\alpha} \rightarrow\left(2 \pi \alpha^{\prime}\right) A_{\alpha}$, $A_{m} \rightarrow\left(2 \pi \alpha^{\prime}\right) A_{m}, \mathcal{W}^{\alpha} \rightarrow\left(2 \pi \alpha^{\prime}\right) \mathcal{W}^{\alpha}$ and $F_{m n} \rightarrow\left(2 \pi \alpha^{\prime}\right) F_{m n}$, the DBI equations (4.32)-(4.34) reduce to the super-YangMills equations of motion (B9)-(B11) with an appropriate dimensional reduction.

\footnotetext{
${ }^{4}$ If we construct the $\kappa$-invariant boundary term which cancels out an $\mathcal{N}=1$ supersymmetry variation of the Green-Schwarz action and turn it into the BRS-invariant boundary term like (3.4) by the method used in [31] (see also Sec. IV.1 in [32]), it must be shown $c_{1}=c_{2}=1$.
}

We note that the ten-dimensional Lorentz covariance is manifestly broken by the boundary term $S_{\mathrm{b}}$ as well as the background coupling $V$. However the obtained DBI equations can be expressed in a covariant form. This implies that our result is consistent with that for a D9-brane.

We expect that we can extend our result so that the BRS invariance should lead to supersymmetric non-Abelian DBI equations of motion on a D $p$-brane. We would like to report this issue in the near future [33].

As an alternative to our study, non-Abelian deformations of the maximally supersymmetric Yang-Mills theory can be specified based on spinorial cohomology [34], which may be closely related to the pure spinor fields in ten- and eleven-dimensional spacetime [35-37]. The structure of higher-derivative invariants in the maximally supersymmetric Yang-Mills theories are studied in [38]. Moreover, in $[39,40]$ the pure spinor superspace formalism is developed, which contains not only (minimal) pure spinor variables but also nonminimal pure spinor variables [41]. This enables us to construct the BRS invariant action for the tendimensional supersymmetric DBI theory. Recently, this offshell action is studied further in $[42,43]$. It is interesting to pursue these issues from the open string point of view.

On the other hand, the classical BRS invariance of a closed pure spinor superstring in a curved background is shown to imply that the background fields satisfy full nonlinear equations of motion for the type II supergravity [44]. This is similar to the result for the classical $\kappa$-invariance of a closed Green-Schwarz superstring [45]. Moreover, recently in [46] the classical $\kappa$-invariance also leads to the generalized type II supergravity equations of motion ${ }^{5}$ whose solutions originally have been found out in the context of integrable deformations of $\mathrm{AdS}_{5} \times \mathrm{S}^{5}$ sigma models [48]. It is also interesting to consider whether the generalization of DBI equations can be derived analogously from the $\kappa$ or BRS invariance of an open superstring.

An immediate task is to clarify contribution of the dilaton superfield to Bianchi identities. In that case we need to investigate closely the DBI equation corresponding to $I_{m n \alpha}=0$ in the super-Yang-Mills theory as we see in Appendix A. This equation is also useful to confirm that our result agrees with the one which comes from the bosonic part of the DBI action.

Finally, it is interesting for us to calculate quantum higher-derivative corrections to our result by analyzing the quantum BRS invariance of the open pure spinor superstring.

\section{ACKNOWLEDGMENTS}

The authors would like to thank Takanori Fujiwara, Yoshifumi Hyakutake and Kentaroh Yoshida for useful comments. S. H. also thanks the Yukawa Institute for

\footnotetext{
${ }^{5}$ See [47] for further investigations based on double field theory.
} 
Theoretical Physics at Kyoto University. Discussions during the workshop YITP-T-18-04 "New Frontiers in String Theory 2018" were useful to complete this work. S. H. and M.S. would like to appreciate the organizers of the conferences "KEK Theory Workshop 2018" held at KEK theory center, Tsukuba, and The 2nd Workshop on "Mathematics and Physics in General Relativity," held at Setsunan University, for their kind hospitality. There, S. H. and M.S. reported the main results in this paper.

\section{APPENDIX A: TEN-DIMENSIONAL $\mathcal{N}=1$ SUPER-YANG-MILLS SPACE}

We will review the ten-dimensional $\mathcal{N}=1$ super-YangMills theory [49]. Introducing a superconnection one-form $A=E^{M} A_{M}$, where $E^{M}$ are supervielbeins and $A_{M}=$ $\left(A_{m}, A_{\alpha}\right)$ are superconnections, we define the gauge supercovariant derivative $\nabla_{M}$ :

$$
\nabla_{m}=\partial_{m}+A_{m}, \quad \nabla_{\alpha}=D_{\alpha}+A_{\alpha},
$$

where $D_{\alpha}$ is the supercovariant derivative defined by

$$
D_{\alpha}=\frac{\partial}{\partial \theta^{\alpha}}+\frac{1}{2}\left(\gamma^{m} \theta\right)_{\alpha} \partial_{m},
$$

which satisfies $\left\{D_{\alpha}, D_{\beta}\right\}=\gamma_{\alpha \beta}^{m} \partial_{m}$. The field strengths $F_{M N}$ are defined by

$$
\left[\nabla_{M}, \nabla_{N}\right\}=T_{M N}^{R} \nabla_{R}+F_{M N},
$$

where $T_{M N}{ }^{R}$ are flat torsion tensors whose components are fixed to zero except for $T_{\alpha \beta}^{m}=\gamma_{\alpha \beta}^{m}$. According to this definition, these field strengths are invariant under the gauge transformations with a superfield parameter $\Omega$ :

$$
\delta A_{m}=\partial_{m} \Omega, \quad \delta A_{\alpha}=D_{\alpha} \Omega .
$$

For the on-shell super-Yang-Mills theory, we might adopt a constraint [35] (see also [36]),

$$
F_{\alpha \beta}=0,
$$

which implies

$$
D_{\alpha} A_{\beta}+D_{\beta} A_{\alpha}+\left\{A_{\alpha}, A_{\beta}\right\}=\gamma_{\alpha \beta}^{m} A_{m} .
$$

If we consider a dimensional reduction to four dimensions, we see that this constraint reduces to the one in the fourdimensional $\mathcal{N}=4$ super-Yang-Mills theory [50].

In the following, let us solve the Bianchi identities represented as

$$
\begin{aligned}
I_{M N R}= & (-1)^{R(M+N)} \nabla_{R} F_{M N}-T_{M N}{ }^{S} F_{S R} \\
& +(-1)^{M(R+N)} \nabla_{N} F_{R M}-(-1)^{R(M+N)} T_{R M}{ }^{S} F_{S N} \\
& +\nabla_{M} F_{N R}-(-1)^{M(N+R)} T_{N R}{ }^{S} F_{S M} .
\end{aligned}
$$

The first identity $I_{\alpha \beta \gamma}=0$ implies

$$
-\gamma_{\alpha \beta}^{m} F_{m \gamma}-\gamma_{\gamma \alpha}^{m} F_{m \beta}-\gamma_{\beta \gamma}^{m} F_{m \alpha}=0 .
$$

Thanks to the Fierz identity, we find that the field strength $F_{m \alpha}$ must take the form of

$$
F_{m \alpha}=-\gamma_{m \alpha \beta} \mathcal{W}^{\beta} .
$$

In other words,

$$
\partial_{m} A_{\alpha}-D_{\alpha} A_{m}+\left[A_{m}, A_{\alpha}\right]=-\gamma_{m \alpha \beta} \mathcal{W}^{\beta} .
$$

Next the second identity $I_{m \alpha \beta}=0$ together with (A9) implies

$$
\gamma_{m \alpha \delta} \nabla_{\beta} \mathcal{W}^{\delta}+\gamma_{m \beta \delta} \nabla_{\alpha} \mathcal{W}^{\delta}-\gamma_{\alpha \beta}^{n} F_{n m}=0 .
$$

Multiplying this by $\gamma_{p}^{\alpha \beta}$, we find that

$$
F_{m n}=\frac{1}{8}\left(\gamma_{m n}\right)_{\alpha}^{\beta} \nabla_{\beta} \mathcal{W}^{\alpha},
$$

which is equivalent to

$$
\nabla_{\alpha} \mathcal{W}^{\beta}=-\frac{1}{4}\left(\gamma^{m n}\right)_{\alpha}^{\beta} F_{m n} .
$$

The third identity $I_{m n \alpha}=0$ implies

$$
\nabla_{\alpha} F_{m n}=\gamma_{n \alpha \beta} \nabla_{m} \mathcal{W}^{\beta}-\gamma_{m \alpha \beta} \nabla_{n} \mathcal{W}^{\beta} .
$$

Taking (A13) into account, (A14) yields the result

$$
\gamma_{\alpha \beta}^{m} \nabla_{m} \mathcal{W}^{\beta}=0 .
$$

Furthermore, multiplying (A15) by $\gamma^{n \gamma \alpha} \nabla_{\gamma}$ we find

$$
\nabla^{m} F_{m n}=-\frac{1}{2} \gamma_{n \alpha \beta}\left\{\mathcal{W}^{\alpha}, \mathcal{W}^{\beta}\right\}
$$

Equations (A16) and (A15) imply the Maxwell equation for the gauge field $\nabla_{m} f^{m n}=0$ and the Dirac equation for the gaugino $\gamma_{\alpha \beta}^{m} \nabla_{m} \xi^{\beta}=0$, respectively.

Finally, the remaining identity $I_{m n p}=0$ implies

$$
\nabla_{m} F_{n p}+\nabla_{n} F_{p m}+\nabla_{p} F_{m n}=0,
$$

and it suggests that $F_{m n}$ is just the curl of a gauge field $A_{m}$ :

$$
F_{m n}=\partial_{m} A_{n}-\partial_{n} A_{m}+\left[A_{m}, A_{n}\right] .
$$

The $\theta$-expansion of these superfields is studied in [51].

\section{APPENDIX B: MASSLESS VERTEX OPERATOR FOR PURE SPINOR OPEN SUPERSTRING}

We present a review of the vertex operators in the open pure spinor superstring [23] (see also [32]). For simplicity, we focus on the left-moving sector only.

We consider a ghost number 1 massless vertex operator given by 


$$
U=\lambda^{\alpha} A_{\alpha}(x, \theta)
$$

where $A_{\alpha}(x, \theta)$ is a spinor superfield. The BRS transformation law is represented as

$$
\begin{aligned}
Q x^{m} & =\frac{1}{2} \lambda \gamma^{m} \theta, \quad Q \theta^{\alpha}=\lambda^{\alpha}, \quad Q d_{\alpha}=-\Pi^{m}\left(\gamma_{m} \lambda\right)_{\alpha}, \\
Q \lambda^{\alpha} & =0, \quad Q \omega_{\alpha}=d_{\alpha},
\end{aligned}
$$

where $Q$ denotes $\delta_{1}$ in Sec. III A. Note that $Q^{2} \omega_{\alpha}=$ $-\Pi^{m}\left(\gamma_{m} \lambda\right)_{\alpha}$ turns out the gauge transformation for $\omega_{\alpha}$. Then the cohomology condition, $Q U=0$ up to the gauge transformation $\delta U=Q \Omega$, implies

$$
D_{\alpha}\left(\gamma_{\text {mnpqr }}\right)^{\alpha \beta} A_{\beta}=0 \text { and } \delta A_{\alpha}=D_{\alpha} \Omega,
$$

where $\Omega(x, \theta)$ is a gauge parameter, the derivative $D_{\alpha}$ is given in (A2).

To derive (B3), we use the pure spinor constraint for the commutative bispinor $\lambda$ :

$$
\lambda^{\alpha} \lambda^{\beta}=\frac{1}{2^{5} 5 !} \gamma_{\mathrm{mnpqr}}^{\alpha \beta}\left(\lambda^{\gamma} \gamma_{\gamma \delta}^{\mathrm{mnpqr}} \lambda^{\delta}\right)
$$

As a result, (B3) is consistent with the super-Yang-Mills equations of motion and the gauge transformations as we have seen in Appendix A.

Next, we derive an integrated vertex operator such as $V=\int d z \mathcal{V}$. Recalling the RNS formulation, $\mathcal{V}$ is given as the anticommutator of the unintegrated vertex operator $U$ and the $b$-ghost. However, in the pure spinor formulation, the reparametrization $b$-ghost is unclear without introducing the nonminimal part $[41] .{ }^{6}$ Fortunately, the above facts can be rephrased in terms of the BRS charge $Q$ as ${ }^{7}$

$$
Q \mathcal{V}=\partial U
$$

We find the vertex operator $\mathcal{V}$ takes the form of

$$
\begin{aligned}
\mathcal{V}= & \partial \theta^{\alpha} A_{\alpha}(x, \theta)+\Pi^{m} A_{m}(x, \theta)+d_{\alpha} \mathcal{W}^{\alpha}(x, \theta) \\
& +\frac{1}{2} N^{m n} F_{m n}(x, \theta),
\end{aligned}
$$

where $N^{m n}=\frac{1}{2} \lambda \gamma^{m n} \omega$ is the ghost Lorentz current. Indeed, since

\footnotetext{
${ }^{6}$ The nonminimal pure spinor formalism extended to the Maxwell background is investigated in [52].

${ }^{7}$ The Jacobi identity implies

$$
\begin{aligned}
Q \mathcal{V} & =[Q,\{\oint d z b, U\}] \\
& =-[U,\{Q, \oint d z b\}]-[\oint d z b,\{U, Q\}]=\partial U
\end{aligned}
$$

since $\{Q, U\}=0,\{Q, b\}=T$ and $[\oint d z T, U]=\partial U$ for the conformal weight zero primary operator $U$.
}

$$
\begin{aligned}
Q \mathcal{V}= & \partial\left(\lambda^{\alpha} A_{\alpha}\right)+\lambda^{\alpha} \partial \theta^{\beta}\left(-D_{\alpha} A_{\beta}-D_{\beta} A_{\alpha}+\gamma_{\alpha \beta}^{m} A_{m}\right) \\
& +\lambda^{\alpha} \Pi^{m}\left(D_{\alpha} A_{m}-\partial_{m} A_{\alpha}-\gamma_{m \alpha \beta} \mathcal{W}^{\beta}\right) \\
& +\lambda^{\alpha} d_{\beta}\left(-D_{\alpha} \mathcal{W}^{\beta}+\frac{1}{4}\left(\gamma^{m n}\right)_{\alpha}^{\beta} F_{m n}\right)+\frac{1}{2} \lambda^{\alpha} N^{m n} D_{\alpha} F_{m n},
\end{aligned}
$$

(B7) implies the following equations:

$$
\begin{gathered}
-D_{\alpha} A_{\beta}-D_{\beta} A_{\alpha}+\gamma_{\alpha \beta}^{m} A_{m}=0, \\
D_{\alpha} A_{m}-\partial_{m} A_{\alpha}-\gamma_{m \alpha \beta} \mathcal{W}^{\beta}=0, \\
-D_{\alpha} \mathcal{W}^{\beta}+\frac{1}{4}\left(\gamma^{m n}\right)_{\alpha}{ }^{\beta} F_{m n}=0, \\
\lambda^{\alpha} \lambda^{\beta}\left(\gamma^{m n}\right)_{\beta}{ }^{\gamma} D_{\alpha} F_{m n}=0 .
\end{gathered}
$$

Equations (B9)-(B11) certainly correspond to the superYang-Mills equations (A6), (A10) and (A13) in the Abelian case, respectively. It follows that superfields $A_{\alpha}$ and $A_{m}$ are spinor and vector gauge fields in the ten-dimensional $\mathcal{N}=$ 1 super-Yang-Mills theory, and that $\mathcal{W}^{\alpha}$ and $F_{m n}$ are spinor and vector field strengths for them. On the other hand, (B12) is satisfied by the pure spinor constraint

$$
\begin{aligned}
\lambda^{\alpha} \lambda^{\beta}\left(\gamma^{m n}\right)_{\beta}{ }^{\gamma} D_{\alpha} F_{m n} & =4 \lambda^{\alpha} \lambda^{\beta} D_{\alpha} D_{\beta} \mathcal{W}^{\gamma} \\
& =2\left(\lambda \gamma^{m} \lambda\right) \partial_{m} \mathcal{W}^{\gamma}=0,
\end{aligned}
$$

where (B11) is used. If (B9) is contracted with $\left(\gamma_{\mathrm{mnpqr}}\right)^{\alpha \beta}$, we obtain the equation of motion for $A_{\alpha}$ in (B3). Contraction of (B9) with $\gamma_{n}^{\alpha \beta}$ also leads to

$$
A_{m}=\frac{1}{8} \gamma_{m}^{\alpha \beta} D_{\alpha} A_{\beta} .
$$

Then the gauge transformation in (B3) turns to $\delta A_{m}=\partial_{m} \Omega$. Similarly contracting (B10) with $\gamma^{m \alpha \gamma}$ implies the equation for $\mathcal{W}^{\alpha}$,

$$
\mathcal{W}^{\beta}=\frac{1}{10} \gamma^{m \alpha \beta}\left(D_{\alpha} A_{m}-\partial_{m} A_{\alpha}\right),
$$

and contracting (B11) with $\left(\gamma^{p q}\right)_{\beta}^{\alpha}$ implies the equation for $F_{m n}$,

$$
F_{m n}=\frac{1}{8}\left(\gamma_{m n}\right)_{\alpha}^{\beta} D_{\beta} \mathcal{W}^{\alpha} .
$$

Furthermore, utilizing (B14), (B10) and (B16), we derive

$$
\begin{aligned}
\partial_{[m} A_{n]} & =-\frac{1}{8} \gamma_{[m}^{\alpha \beta} D_{\alpha}\left(\partial_{n]} A_{\beta}\right)=-\frac{1}{8} \gamma_{[m}^{\alpha \beta} D_{\alpha}\left(D_{\beta} A_{n]}-\left(\gamma_{n]} \mathcal{W}\right)_{\beta}\right) \\
& =\frac{1}{8}\left(\gamma_{m n}\right)_{\beta}^{\alpha} D_{\alpha} \mathcal{W}^{\beta}=F_{m n} .
\end{aligned}
$$

Besides, this equation together with (B10) implies 


$$
D_{\alpha} F_{m n}=\partial_{[m} D_{|\alpha|} A_{n]}=\partial_{[m}\left(\gamma_{n]} \mathcal{W}\right)_{\alpha} .
$$

(B17) and (B18) certainly correspond to remaining Bianchi identities (A18) and (A14) for the Abelian case, respectively.

\section{APPENDIX C: BRS CHARGE CONSERVATION}

We will derive the supersymmetric DBI equations by modifying the method used in [24] to include the Dirichlet components.
We require that the general variation $\delta\left(S_{0}+S_{\mathrm{b}}+V\right)$ vanishes. This leads to boundary conditions in the presence of background superfields. Under these conditions, it is shown that the BRS charge conservation implies superfield equations for DBI fields.

Let us begin to examine a general variation of the worldsheet action $S_{0}$ in (2.1), its ten-dimensional $\mathcal{N}=1$ supersymmetry counterterm $S_{\mathrm{b}}$ in (3.4) and the background coupling $V$ in (4.1). We find that variations $\delta\left(S_{0}+S_{\mathrm{b}}\right)$ and $\delta V$ may be expressed as

$$
\begin{aligned}
& \delta\left(S_{0}+S_{\mathrm{b}}\right)= \frac{1}{2 \pi \alpha^{\prime}} \int \mathrm{d} \tau\left\{\delta \theta_{+}^{\alpha}\left[\frac{1}{2} d_{\alpha}^{-}+\Pi_{+}^{\mu}\left(\gamma_{\mu} \theta_{-}\right)_{\alpha}+\tilde{\Pi}_{+}^{i}\left(\gamma_{i} \theta_{-}\right)_{\alpha}-y_{i}\left(\gamma^{i} \dot{\theta}_{+}\right)_{\alpha}+\frac{1}{6}\left(\theta_{-} \gamma^{m} \dot{\theta}_{-}\right)\left(\gamma_{m} \theta_{-}\right)_{\alpha}\right]\right. \\
&+\delta \theta_{-}^{\alpha}\left[\frac{1}{2}\left(1-c_{1}\right) \Delta_{\alpha}^{+}-\frac{1}{6}\left(\theta_{-} \gamma^{m} \dot{\theta}_{+}\right)\left(\gamma_{m} \theta_{-}\right)_{\alpha}\right]-\delta y_{+}^{\mu}\left[\tilde{\Pi}_{-\mu}-\frac{1}{2}\left(\theta_{-} \dot{\gamma}_{\mu} \dot{\theta}_{+}\right)\right] \\
&\left.+\delta \tilde{\Pi}_{+}^{i} y_{i}+\frac{1}{2} c_{1} \delta \Delta_{\alpha}^{+} \theta_{-}^{\alpha}+\frac{1}{2}\left(c_{2}-1\right) \omega_{\alpha}^{+} \delta \lambda_{-}^{\alpha}+\frac{1}{2} c_{2} \delta \omega_{\alpha}^{+} \lambda_{-}^{\alpha}-\frac{1}{2} \omega_{\alpha}^{-} \delta \lambda_{+}^{\alpha}\right\}, \\
& \delta V=\frac{1}{2 \pi \alpha^{\prime}} \int \mathrm{d} \tau\left\{\delta \theta_{+}^{\alpha}\left[\dot{\theta}_{+}^{\beta}\left(\gamma_{\alpha \beta}^{\mu} A_{\mu}-D_{\alpha} A_{\beta}-D_{\beta} A_{\alpha}\right)+\Pi_{+}^{\mu}\left(D_{\alpha} A_{\mu}-\partial_{\mu} A_{\alpha}\right)+\tilde{\Pi}_{+}^{i} D_{\alpha} A_{i}-\frac{1}{2} \Delta_{\beta}^{+} D_{\alpha} \mathcal{W}^{\beta}+\frac{1}{4} D_{\alpha}\left(N_{+} \not{F}\right)\right]\right. \\
&+\delta y_{+}^{\mu}\left[\dot{\theta}_{+}^{\alpha}\left(\partial_{\mu} A_{\alpha}-D_{\alpha} A_{\mu}\right)+\Pi_{+}^{\nu}\left(\partial_{\mu} A_{\nu}-\partial_{\nu} A_{\mu}\right)+\tilde{\Pi}_{+}^{i} \partial_{\mu} A_{i}+\frac{1}{2} \Delta_{\alpha}^{+} \partial_{\mu} \mathcal{W}^{\alpha}+\frac{1}{4} \partial_{\mu}\left(N_{+} \not{F}\right)\right]+\delta \tilde{\Pi}_{+}^{i} A_{i} \\
&\left.+\frac{1}{2} \delta \Delta_{\alpha}^{+} \mathcal{W}^{\alpha}+\frac{1}{8} \delta \lambda_{+}^{\alpha} \mathscr{F}^{\beta}{ }_{\alpha} \omega_{\beta}^{+}+\frac{1}{8} \lambda_{+}^{\alpha} \mathscr{F}_{\alpha}^{\beta} \delta \omega_{\beta}^{+}\right\},
\end{aligned}
$$

where $\delta y^{\mu}$ defined by

$$
\delta y_{+}^{\mu}=\delta x^{\mu}+\frac{1}{2}\left(\theta_{+} \gamma^{\mu} \delta \theta_{+}\right)
$$

is invariant under the $\mathcal{N}=1$ supersymmetry. We also see that $\delta\left(S_{0}+S_{\mathrm{b}}\right) / \delta y_{i} \mid=0$ as mentioned in Sec. III.

To obtain boundary conditions from $\delta\left(S_{0}+S_{\mathrm{b}}+V\right)=0$, first we focus on the terms with $\delta \Delta_{\alpha}^{+}$and $\delta \omega_{\alpha}^{+}$, and derive

$$
\theta_{-}^{\alpha}=-\frac{1}{c_{1}} \mathcal{W}^{\alpha}, \quad \lambda_{-}^{\alpha}=-\frac{1}{4 c_{2}} \lambda_{+}^{\beta} F_{\beta}^{\alpha} .
$$

They also lead to

$$
\begin{aligned}
\dot{\theta}_{-}^{\alpha} & =-\frac{1}{c_{1}}\left(\Pi_{+}^{\mu} \partial_{\mu} \mathcal{W}^{\alpha}+\dot{\theta}_{+}^{\beta} D_{\beta} \mathcal{W}^{\alpha}\right), \\
\delta \theta_{-}^{\alpha} & =-\frac{1}{c_{1}}\left(\delta y_{+}^{\mu} \partial_{\mu} \mathcal{W}^{\alpha}+\delta \theta_{+}^{\beta} D_{\beta} \mathcal{W}^{\alpha}\right), \\
\delta \lambda_{-}^{\alpha} & =-\frac{1}{4 c_{2}} \delta \lambda_{+}^{\beta} \not^{\alpha}{ }_{\beta}-\frac{1}{4 c_{2}}\left(\lambda_{+}^{\beta} \delta y_{+}^{\mu} \partial_{\mu} \not^{\alpha}{ }_{\beta}+\lambda_{+}^{\beta} \delta \theta_{+}^{\gamma} D_{\gamma} F^{\alpha}{ }_{\beta}\right) .
\end{aligned}
$$

Next, examining the terms with $\delta \lambda_{+}^{\alpha}$ in $\delta\left(S_{0}+S_{\mathrm{b}}+V\right)$ we find

$$
\omega_{\alpha}^{-}=\frac{1}{4 c_{2}} \omega_{\beta}^{+} F_{\alpha}^{\beta} .
$$

Boundary conditions for $\lambda_{-}^{\alpha}$ in (C4) and $\omega_{\alpha}^{-}$in (C6) are consistent with the ghost number charge conservation $\lambda^{\alpha} \omega_{\alpha}\left|=\hat{\lambda}^{\alpha} \hat{\omega}_{\alpha}\right|$, where "|" means "evaluated at the boundary." On the other hand, we can eliminate the terms with $\delta \tilde{\Pi}_{+}^{i}$ in $\delta\left(S_{0}+S_{\mathrm{b}}+V\right)$ by the identification (4.14). After substituting the above conditions into $\delta\left(S_{0}+S_{\mathrm{b}}+V\right)=0$, we examine the terms with $\delta y_{+}^{\mu}$ and $\delta \theta_{+}^{\alpha}$. They lead to complicated boundary conditions: 


$$
\begin{gathered}
\tilde{\Pi}_{-\mu}=\dot{\theta}_{+}^{\alpha}\left(\partial_{\mu} A_{\alpha}-D_{\alpha} A_{\mu}+\frac{1}{2 c_{1}}\left(\gamma_{\mu} \mathcal{W}\right)_{\alpha}+\frac{1}{6 c_{1}^{3}}\left(\gamma^{m} \mathcal{W}\right)_{\alpha}\left(\mathcal{W} \gamma_{m} \partial_{\mu} \mathcal{W}\right)\right) \\
+\Pi_{+}^{\nu}\left(\partial_{\mu} A_{\nu}-\partial_{\nu} A_{\mu}\right)+\tilde{\Pi}_{+}^{i} \partial_{\mu} A_{i}+\frac{1}{2 c_{1}} \Delta_{\alpha}^{+} \partial_{\mu} \mathcal{W}^{\alpha}+\frac{1}{4 c_{2}} \partial_{\mu}\left(N_{+} \not{F}\right), \\
\frac{1}{2} d_{\alpha}^{-}=\dot{\theta}_{+}^{\beta}\left(D_{\alpha} A_{\beta}+D_{\beta} A_{\alpha}-\gamma_{\alpha \beta}^{m} A_{m}-\frac{1}{6 c_{1}^{3}}\left(\mathcal{W} \gamma^{m} D_{\beta} \mathcal{W}\right)\left(\gamma_{m} \mathcal{W}\right)_{\alpha}-\frac{1}{6 c_{1}^{3}}\left(\mathcal{W} \gamma^{m} D_{\alpha} \mathcal{W}\right)\left(\gamma_{m} \mathcal{W}\right)_{\beta}\right) \\
+\Pi_{+}^{\mu}\left(\partial_{\mu} A_{\alpha}-D_{\alpha} A_{\mu}+\frac{1}{c_{1}}\left(\gamma_{\mu} \mathcal{W}\right)_{\alpha}+\frac{1}{6 c_{1}^{3}}\left(\mathcal{W} \gamma^{m} \partial_{\mu} \mathcal{W}\right)\left(\gamma_{m} \mathcal{W}\right)_{\alpha}\right) \\
+\tilde{\Pi}_{+}^{i}\left(\frac{1}{c_{1}}\left(\gamma_{i} \mathcal{W}\right)_{\alpha}-D_{\alpha} A_{i}\right)+\frac{1}{2 c_{1}} \Delta_{\beta}^{+} D_{\alpha} \mathcal{W}^{\beta}-\frac{1}{4 c_{2}} D_{\alpha}\left(N_{+} \not{F}\right) .
\end{gathered}
$$

Equation (C7) is regarded as a modified Neumann boundary condition. Boundary conditions for $\omega_{\alpha}^{-}$in (C6) and $d_{\alpha}^{-}$in (C8) must be consistent with the BRS transformation $\delta_{Q} \omega_{\alpha}^{-}=d_{\alpha}^{-}$up to the $\Lambda$-gauge transformation in Sec. II. In the following discussion, we will absorb $c_{1}$ and $c_{2}$ by rescaling $\mathcal{W}^{\alpha} \rightarrow c_{1} \mathcal{W}^{\alpha}$ and $\not^{\beta}{ }_{\alpha} \rightarrow c_{2} \not^{\beta}{ }_{\alpha}$.

To extract DBI equations, we impose the following relation for BRS currents:

$$
\lambda^{\alpha} d_{\alpha}\left|=\hat{\lambda}^{\alpha} \hat{d}_{\alpha}\right|
$$

which implies BRS charge conservation

$$
0=\partial_{\tau} Q_{\mathrm{total}}=\int \mathrm{d} \sigma \partial_{\tau}\left(j_{\mathrm{BRS}}^{\tau}\right)=\int \mathrm{d} \sigma \partial_{\sigma}\left(j_{\mathrm{BRS}}^{\sigma}\right)=\int \mathrm{d} \sigma \partial_{\sigma}\left(j_{\mathrm{BRS}}^{z}-j_{\mathrm{BRS}}^{\bar{z}}\right)=\left(\lambda^{\alpha} d_{\alpha}-\hat{\lambda}^{\alpha} \hat{d}_{\alpha}\right) \mid .
$$

Then we assume the Dirichlet boundary condition

$$
\Pi_{-i}=-\Pi_{+}^{\mu} \partial_{\mu} A_{i}-\dot{\theta}_{+}^{\alpha} D_{\alpha} A_{i}+\frac{1}{2 c_{1}}\left(\dot{\theta}_{+} \gamma_{i} \mathcal{W}\right) .
$$

This is parallel with the Neumann boundary condition in (C7) and just the derivation of the identification (4.14) with respect to the time-coordinate $\tau$.

Under these boundary conditions (C4), (C7), (C8) and (C11), the BRS charge conservation (C9) implies

$$
\begin{aligned}
0= & \hat{\lambda}^{\alpha} \hat{d}_{\alpha}-\lambda^{\alpha} d_{\alpha} \\
= & \frac{1}{2} \lambda_{+}^{\alpha} d_{\alpha}^{-}+\frac{1}{2} \lambda_{-}^{\alpha} \Delta_{\alpha}^{+}-\frac{1}{2}\left(\lambda_{-} \gamma_{\mu} \theta_{-}\right) \tilde{\Pi}_{-}^{\mu}-\frac{1}{2}\left(\lambda_{-} \gamma_{i} \theta_{-}\right) \Pi_{-}^{i}-\frac{1}{4}\left(\lambda_{-} \gamma^{m} \theta_{-}\right)\left(\theta_{-} \gamma_{m} \dot{\theta}_{+}\right) \\
= & \lambda_{+}^{\alpha} \dot{\theta}_{+}^{\beta}\left[D_{\alpha} A_{\beta}+D_{\beta} A_{\alpha}-\gamma_{\alpha \beta}^{m} A_{m}-\frac{1}{6}\left(\mathcal{W} \gamma^{m} D_{\beta} \mathcal{W}\right)\left(\gamma_{m} \mathcal{W}\right)_{\alpha}-\frac{1}{6}\left(\mathcal{W} \gamma^{m} D_{\alpha} \mathcal{W}\right)\left(\gamma_{m} \mathcal{W}\right)_{\beta}\right. \\
& \left.+\frac{1}{8} \not \gamma_{\alpha}^{\gamma}\left(\gamma^{m} \mathcal{W}\right)_{\gamma}\left\{\partial_{m} A_{\beta}-D_{\beta} A_{m}+\left(\gamma_{m} \mathcal{W}\right)_{\beta}+\frac{1}{6}\left(\gamma^{n} \mathcal{W}\right)_{\beta}\left(\mathcal{W} \gamma_{n} \partial_{m} \mathcal{W}\right)\right\}\right] \\
& +\lambda_{+}^{\alpha} \Pi_{+}^{\mu}\left[\partial_{\mu} A_{\alpha}-D_{\alpha} A_{\mu}+\left(\gamma_{\mu} \mathcal{W}\right)_{\alpha}+\frac{1}{6}\left(\mathcal{W} \gamma^{m} \partial_{\mu} \mathcal{W}\right)\left(\gamma_{m} \mathcal{W}\right)_{\alpha}+\frac{1}{8} \not^{\beta}{ }_{\alpha}\left(\gamma^{n} \mathcal{W}\right)_{\beta}\left(\partial_{\mu} A_{n}-\partial_{n} A_{\mu}\right)\right] \\
& +\lambda_{+}^{\alpha} \tilde{\Pi}_{+}^{i}\left[-D_{\alpha} A_{i}+\left(\gamma_{i} \mathcal{W}\right)_{\alpha}-\frac{1}{8} \not^{\beta}{ }_{\alpha}\left(\gamma^{\mu} \mathcal{W}\right)_{\beta} \partial_{\mu} A_{i}\right]+\frac{1}{2} \lambda_{+}^{\alpha} \Delta_{\beta}^{+}\left[D_{\alpha} \mathcal{W}^{\beta}-\frac{1}{4} \not^{\beta}{ }_{\alpha}+\frac{1}{8} \not^{\gamma}{ }_{\alpha}\left(\gamma^{\mu} \mathcal{W}\right)_{\gamma} \partial_{\mu} \mathcal{W}^{\beta}\right] \\
& -\frac{1}{4} \lambda_{+}^{\alpha} N_{+\gamma}^{\beta}\left[D_{\alpha} \not^{\gamma \gamma}{ }_{\beta}+\frac{1}{8} \not^{\delta}{ }_{\alpha}\left(\gamma^{\mu} \mathcal{W}\right)_{\delta} \partial_{\mu} \boldsymbol{F}^{\gamma}{ }_{\beta}\right] .
\end{aligned}
$$

Finally, we find that, to eliminate this expression, (4.31), (4.27), (4.19), (4.20) and (4.26) should be required, as expected. The first four equations are supersymmetric DBI equations of motion on a D $p$-brane, and the last one is the pure spinor constraint. 
[1] E. S. Fradkin and A. A. Tseytlin, Nonlinear electrodynamics from quantized strings, Phys. Lett. 163B, 123 (1985); A. Abouelsaood, C. G. Callan, Jr., C. R. Nappi, and S. A. Yost, Open strings in background fields, Nucl. Phys. B280, 599 (1987); R. G. Leigh, Dirac-Born-Infeld action from Dirichlet sigma model, Mod. Phys. Lett. A 04, 2767 (1989).

[2] E. Bergshoeff, E. Sezgin, C. N. Pope, and P. K. Townsend, The Born-Infeld action from conformal invariance of the open superstring, Phys. Lett. B 188, 70 (1987); O. D. Andreev and A. A. Tseytlin, Partition function representation for the open superstring effective action: Cancellation of Mobius infinities and derivative corrections to BornInfeld Lagrangian, Nucl. Phys. B311, 205 (1988); C. G. Callan, Jr., C. Lovelace, C. R. Nappi, and S. A. Yost, Loop corrections to superstring equations of motion, Nucl. Phys. B308, 221 (1988).

[3] M. Aganagic, C. Popescu, and J. H. Schwarz, D-Brane actions with local kappa symmetry, Phys. Lett. B 393, 311 (1997); M. Cederwall, A. von Gussich, B. E. W. Nilsson, and A. Westerberg, The Dirichlet super three-brane in tendimensional type IIB supergravity, Nucl. Phys. B490, 163 (1997); M. Cederwall, A. von Gussich, B. E. W. Nilsson, P. Sundell, and A. Westerberg, The Dirichlet super $p$-branes in ten-dimensional type IIA and IIB supergravity, Nucl. Phys. B490, 179 (1997); E. Bergshoeff and P. K. Townsend, Super D-branes, Nucl. Phys. B490, 145 (1997).

[4] V. Akulov, I. A. Bandos, W. Kummer, and V. Zima, $D=10$ Dirichlet super-9-brane, Nucl. Phys. B527, 61 (1998).

[5] P. S. Howe and E. Sezgin, Superbranes, Phys. Lett. B 390, 133 (1997); P. S. Howe, O. Raetzel, and E. Sezgin, On brane actions and superembeddings, J. High Energy Phys. 08 (1998) 011; D. P. Sorokin, Superbranes and superembeddings, Phys. Rep. 329, 1 (2000).

[6] C. S. Chu, P. S. Howe, and E. Sezgin, Strings and D-branes with boundaries, Phys. Lett. B 428, 59 (1998).

[7] P. S. Howe, U. Lindström, and L. Wulff, Superstrings with boundary fermions, J. High Energy Phys. 08 (2005) 041; On the covariance of the Dirac-Born-Infeld-Myers action, J. High Energy Phys. 02 (2007) 070; Kappa-symmetry for coincident D-branes, J. High Energy Phys. 09 (2007) 010.

[8] E. Bergshoeff, M. Rakowski, and E. Sezgin, Higher derivative Super-Yang-Mills theories, Phys. Lett. B 185, 371 (1987).

[9] A. A. Tseytlin, On non-Abelian generalization of the BornInfeld action in string theory, Nucl. Phys. B501, 41 (1997).

[10] W. Taylor and M. Van Raamsdonk, Multiple D $p$-branes in weak background fields, Nucl. Phys. B573, 703 (2000).

[11] S. V. Ketov, $N=1$ and $N=2$ Supersymmetric non-Abelian Born-Infeld actions from superspace, Phys. Lett. B 491, 207 (2000).

[12] A. Sevrin, J. Troost, and W. Troost, The non-Abelian BornInfeld action at order $F^{6}$, Nucl. Phys. B603, 389 (2001).

[13] E. A. Bergshoeff, M. de Roo, and A. Sevrin, Non-Abelian Born-Infeld and kappa-symmetry, J. Math. Phys. (N.Y.) 42, 2872 (2001); E. A. Bergshoeff, A. Bilal, M. de Roo, and A. Sevrin, Supersymmetric non-Abelian Born-Infeld revisited, J. High Energy Phys. 07 (2001) 029.

[14] A. Refolli, A. Santambrogio, N. Terzi, and D. Zanon, $F^{5}$ contributions to the non-Abelian Born-Infeld action from a supersymmetric Yang-Mills five-point function, Nucl. Phys. B613, 64 (2001).

[15] D. P. Sorokin, Coincident (super) D $p$-branes of codimension one, J. High Energy Phys. 08 (2001) 022.

[16] P. Koerber and A. Sevrin, The non-Abelian Born-Infeld action through order $\alpha^{\prime 3}$, J. High Energy Phys. 10 (2001) 003; The non-Abelian D-brane effective action through order $\alpha^{\prime 4}$, J. High Energy Phys. 10 (2002) 046.

[17] A. Collinucci, M. de Roo, and M. G. C. Eenink, Supersymmetric Yang-Mills theory at order $\alpha^{\prime 3}$, J. High Energy Phys. 06 (2002) 024.

[18] R. Medina, F. T. Brandt, and F. R. Machado, The open superstring 5-point amplitude revisited, J. High Energy Phys. 07 (2002) 071.

[19] J. M. Drummond, P. S. Howe, and U. Lindstrom, Kappa symmetric non-Abelian Born-Infeld actions in three dimensions, Classical Quantum Gravity 19, 6477 (2002).

[20] J. M. Drummond, P. J. Heslop, P. S. Howe, and S. F. Kerstan, Integral invariants in $N=4 \mathrm{SYM}$ and the effective action for coincident D-branes, J. High Energy Phys. 08 (2003) 016.

[21] O. Chandia and R. Medina, 4-point effective actions in open and closed superstring theory, J. High Energy Phys. 11 (2003) 003.

[22] L. A. Barreiro and R. Medina, 5-field terms in the open superstring effective action, J. High Energy Phys. 03 (2005) 055 .

[23] N. Berkovits, Super-Poincaré covariant quantization of the superstring, J. High Energy Phys. 04 (2000) 018.

[24] N. Berkovits and V. Pershin, Supersymmetric Born-Infeld from the pure spinor formalism of the open superstring, J. High Energy Phys. 01 (2003) 023.

[25] S. F. Kerstan, Supersymmetric Born-Infeld from the D9brane, Classical Quantum Gravity 19, 4525 (2002).

[26] R. Schiappa and N. Wyllard, D-brane boundary state in the pure spinor superstring, J. High Energy Phys. 07 (2005) 070 .

[27] L. Anguelova and P. A. Grassi, Super D-branes from BRST symmetry, J. High Energy Phys. 11 (2003) 010.

[28] P. Mukhopadhyay, On D-brane boundary state analysis in pure spinor formalism, J. High Energy Phys. 03 (2006) 066.

[29] S. Hanazawa and M. Sakaguchi, D-branes from pure spinor superstring in $\mathrm{AdS}_{5} \times \mathrm{S}^{5}$ background, Nucl. Phys. B914, 234 (2017).

[30] S. Hanazawa and M. Sakaguchi, Non-commutative M-branes from open pure spinor supermembrane, Nucl. Phys. B927, 566 (2018).

[31] I. Oda and M. Tonin, On the Berkovits covariant quantization of GS superstring, Phys. Lett. B 520, 398 (2001).

[32] N. Berkovits, ICTP lectures on covariant quantization of the superstring, ICTP Lect. Notes Ser. 13, 57 (2003).

[33] S. Hanazawa and M. Sakaguchi (in preparation).

[34] M. Cederwall, B. E. W. Nilsson, and D. Tsimpis, The structure of maximally supersymmetric Yang-Mills theory: Constraining higher-order corrections, J. High Energy Phys. 06 (2001) 034; $D=10$ super Yang-Mills at $O\left(\alpha^{\prime 2}\right)$, J. High Energy Phys. 07 (2001) 042; Spinorial cohomology and maximally supersymmetric theories, J. High Energy Phys. 02 (2002) 009. 
[35] B. E. W. Nilsson, Off-shell fields for ten-dimensional supersymmetric Yang-Mills theory, Gotenburg preprint 81-6, 1981 (unpublished); Pure spinors as auxiliary fields in the ten-dimensional supersymmetric Yang-Mills theory, Classical Quantum Gravity 3, L41 (1986).

[36] P. S. Howe, Pure spinor lines in superspace and tendimensional supersymmetric theories, Phys. Lett. B 258, 141 (1991).

[37] P. S. Howe, Pure spinors, function superspace and supergravity theories in ten and eleven dimensions, Phys. Lett. B 273, 90 (1991).

[38] P. S. Howe, U. Lindström, and L. Wulff, $D=10$ supersymmetric Yang-Mills theory at $\alpha^{\prime 4}$, J. High Energy Phys. 07 (2010) 028; G. Bossard, P. S. Howe, U. Lindström, K. S. Stelle, and L. Wulff, Integral invariants in maximally supersymmetric Yang-Mills theories, J. High Energy Phys. 05 (2011) 021.

[39] M. Cederwall and A. Karlsson, Pure spinor superfields and Born-Infeld theory, J. High Energy Phys. 11 (2011) 134.

[40] M. Cederwall, Pure spinor superfields an overview, Springer Proc. Phys. 153, 61 (2014).

[41] N. Berkovits, Pure spinor formalism as an $N=2$ topological string, J. High Energy Phys. 10 (2005) 089; N. Berkovits and N. Nekrasov, Multiloop superstring amplitudes from nonminimal pure spinor formalism, J. High Energy Phys. 12 (2006) 029.

[42] C. M. Chang, Y. H. Lin, Y. Wang, and X. Yin, Deformations with maximal supersymmetries part 2: Off-shell formulation, J. High Energy Phys. 04 (2016) 171.

[43] N. Berkovits and M. Guillen, Equations of motion from Cederwall's pure spinor superspace actions, J. High Energy Phys. 08 (2018) 033.

[44] N. Berkovits and P. S. Howe, Ten-dimensional supergravity constraints from the pure spinor formalism for the superstring, Nucl. Phys. B635, 75 (2002).
[45] M. Grisaru, P. S. Howe, L. Mezincescu, B. E. W. Nilsson, and P. K. Townsend, $N=2$ superstrings in a supergravity background, Phys. Lett. 162B, 116 (1985).

[46] A. A. Tseytlin and L. Wulff, Kappa-symmetry of superstring sigma model and generalized 10d supergravity equations, J. High Energy Phys. 06 (2016) 174.

[47] Y. Sakatani, S. Uehara, and K. Yoshida, Generalized gravity from modified DFT, J. High Energy Phys. 04 (2017) 123; A. Baguet, M. Magro, and H. Samtleben, Generalized IIB supergravity from exceptional field theory, J. High Energy Phys. 03 (2017) 100; J. Sakamoto, Y. Sakatani, and K. Yoshida, Weyl invariance for generalized supergravity backgrounds from the doubled formalism, Prog. Theor. Exp. Phys. 17, 053 B07 (2017).

[48] G. Arutyunov, S. Frolov, B. Hoare, R. Roiban, and A. A. Tseytlin, Scale invariance of the $\eta$-deformed $\mathrm{AdS}_{5} \times S^{5}$ superstring, T-duality and modified type II equations, Nucl. Phys. B903, 262 (2016).

[49] W. Siegel, Superfields in higher dimensional spacetime, Phys. Lett. 80B, 220 (1979); E. Witten, Twistor-like transform in ten dimensions, Nucl. Phys. B266, 245 (1986).

[50] M. Sohnius, Bianchi identities for supersymmetric theories, Nucl. Phys. B136, 461 (1978).

[51] J. P. Harnad and S. Shnider, Constraints and field equations for ten-dimensional super-Yang-Mills theory, Commun. Math. Phys. 106, 183 (1986); H. Ooguri, J. Rahmfeld, H. Robins, and J. Tannenhauser, Holography in superspace, J. High Energy Phys. 07 (2000) 045; P. A. Grassi and L. Tamassia, Vertex operators for closed superstrings, J. High Energy Phys. 07 (2004) 071; G. Policastro and D. Tsimpis, $R^{4}$, purified, Classical Quantum Gravity 23, 4753 (2006).

[52] I. Bakhmatov and N. Berkovits, Pure spinor $b$-ghost in a super-Maxwell background, J. High Energy Phys. 11 (2013) 214. 